\title{
Comparison of Mean Variance Like Strategies for Optimal Asset Allocation Problems *
}

\author{
J. Wang ${ }^{\dagger} \quad$ P.A. Forsyth ${ }^{\ddagger}$
}

March 14, 2011

\begin{abstract}
We determine the optimal dynamic investment policy for a mean quadratic variation objective function by numerical solution of a nonlinear Hamilton-Jacobi-Bellman (HJB) partial differential equation (PDE). We compare the efficient frontiers and optimal investment policies for three mean variance like strategies: pre-commitment mean variance, time-consistent mean variance, and mean quadratic variation, assuming realistic investment constraints (e.g. no bankruptcy, finite shorting, borrowing). When the investment policy is constrained, the efficient frontiers for all three objective functions are similar, but the optimal policies are quite different.
\end{abstract}

Keywords: Mean quadratic variation investment policy, mean variance asset allocation, HJB equation, optimal control

JEL Classification: C63, G11

AMS Classification $65 \mathrm{~N} 06,93 \mathrm{C} 20$

\section{Introduction}

In this paper, we consider optimal continuous time asset allocation using mean variance like strategies. This contrasts with the classic power law or exponential utility function approach [24].

Mean variance strategies have a simple intuitive interpretation, which is appealing to both individual investors and institutions. There has been considerable recent interest in continuous time mean variance asset allocation [32, 21, 25, 20, 6, 11, 31, 18, 19, 29. However, the optimal strategy in these papers was based on a pre-commitment strategy which is not time-consistent [7, 5].

Although the pre-commitment strategy is optimal in the sense of maximizing the expected return for a given standard deviation, this may not always be economically sensible. A realworld investor experiences only one of many possible stochastic paths [22], hence it is not clear that a strategy which is optimal in an average sense over many stochastic paths is appropriate. In

${ }^{*}$ This work was supported by a grant from Tata Consultancy Services and the Natural Sciences and Engineering Research Council of Canada.

${ }^{\dagger}$ David R. Cheriton School of Computer Science, University of Waterloo, Waterloo ON, Canada N2L 3G1 e-mail: j27wang@uwaterloo.ca

${ }^{\ddagger}$ David R. Cheriton School of Computer Science, University of Waterloo, Waterloo ON, Canada N2L 3G1 e-mail: paforsyt@uwaterloo.ca 
addition, the optimal strategy computed from the pre-commitment objective function assumes that the stochastic parameters are known at the beginning of the investment horizon, and do not change over the investment period. In practice, of course, one would normally recompute the investment strategy based on the most recent available data.

For these reasons, a time-consistent form of mean variance asset allocation has been suggested recently [7, 5, 30]. We may view the time-consistent strategy as a pre-commitment policy with a time-consistent constraint [30].

Another criticism of both time-consistent and pre-commitment strategies is that the risk is only measured in terms of the standard deviation at the end of the investment period. In an effort to provide a more direct control over risk during the investment period, a mean quadratic variation objective function has been proposed in [9, 16].

This article is the third in a series. In [29], we developed numerical techniques for determining the optimal controls for pre-commitment mean variance strategies. The methods in [29] allowed us to apply realistic constraints to the control policies. In [30], we developed numerical methods for solution of the time-consistent formulation of the mean-variance strategy [5]. The methods in [30] also allowed us to apply constraints to the control policies.

In this article, we develop numerical methods for solution of the mean quadratic variation policy, again for the case of constrained controls. We also present a comparison of pre-commitment, time consistent and mean quadratic variation strategies, for two typical asset allocation problems. We emphasize here that we use numerical techniques which allow us to apply realistic constraints (e.g. no bankruptcy, finite borrowing and shorting), on the investment policies. This is in contrast to the analytic approaches used previously [32, 21, 6, 7].

We first consider the optimal investment policy for the holder of a pension plan, who can dynamically allocate his wealth between a risk-free asset and a risky asset. We will also consider the case where the pension plan holder desires to maximize the wealth-to-income ratio, in the case where the plan holder's salary is stochastic [10].

The main results in this paper are

- We formulate the optimal investment policy for the mean quadratic variation problem as a nonlinear Hamilton-Jacobi-Bellman (HJB) partial differential equation (PDE). We extend the numerical methods in [29, 30] to handle this case.

- We give numerical results comparing all three investment policies: pre-commitment mean variance, time-consistent mean variance, and mean quadratic variation. In the case where analytic solutions are available, our numerical results agree with the analytic solutions. In the case where typical constraints are applied to the investment strategy, the efficient frontiers for all three objective functions are very similar. However, the investment policies are quite different.

These results show that, in deciding which objective function is appropriate for a given economic problem, it is not sufficient to simply examine the efficient frontiers. Instead, the actual investment policies need to be studied in order to determine if a particular strategy is applicable to specific investment objectives. 


\section{Dynamic Strategies}

In this paper, we first consider the problem of determining the mean variance like strategies for a pension plan. It is common to write the efficient frontier in terms of the investor's final wealth. We will refer to this problem in the following as the wealth case.

Suppose there are two assets in the market: one is risk free (e.g. a government bond) and the other is risky (e.g. a stock index). The risky asset $S$ follows the stochastic process

$$
d S=(r+\xi \sigma) S d t+\sigma S d Z_{1},
$$

where $d Z_{1}$ is the increment of a Wiener process, $\sigma$ is volatility, $r$ is the interest rate, and $\xi$ is the market price of risk (or Sharpe ratio). The stock drift rate can then be defined as $\mu_{S}=r+\xi \sigma$. We specify the drift rate of the stock in terms of the market price of risk $\xi$, to be consistent with [10]. This also allows us to compare results obtained by varying $\sigma$, while keeping $\xi$ constant, in addition to varying $\sigma$, while keeping $\mu_{S}$ constant.

Suppose that the plan member continuously pays into the pension plan at a constant contribution rate $\pi \geq 0$ in the unit time. Let $W(t)$ denote the wealth accumulated in the pension plan at time t, let $p$ denote the proportion of this wealth invested in the risky asset $S$, and let $(1-p)$ denote the fraction of wealth invested in the risk free asset. Then,

$$
\begin{aligned}
d W & =[(r+p \xi \sigma) W+\pi] d t+p \sigma W d Z_{1}, \\
W(t=0) & =\hat{w}_{0} \geq 0 .
\end{aligned}
$$

Define,

$$
\begin{aligned}
E[\cdot] & : \text { expectation operator, } \\
\operatorname{Var}[\cdot] & : \text { variance operator, } \\
\operatorname{Std}[\cdot] & : \text { standard deviation operator, } \\
E_{t, w}[\cdot], \operatorname{Var}_{t, w}[\cdot] \text { or } \operatorname{Std}_{t, w}[\cdot] & : E[\cdot \mid W(t)=w], \operatorname{Var}[\cdot \mid W(t)=w] \text { or } \operatorname{Std}[\cdot \mid W(t)=w] \\
& \text { when sitting at time } t, \\
E_{t, w}^{p}[\cdot], \operatorname{Var}_{t, w}^{p}[\cdot] \text { or } \operatorname{Std}_{t, w}^{p}[\cdot] & : E_{t, w}[\cdot], \operatorname{Var}_{t, w}[\cdot] \text { or } \operatorname{Std}_{t, w}[\cdot], \text { where } p(s, W(s)), s \geq t, \\
& \text { is the policy along path } W(t) \text { from stochastic process } 2.2 .
\end{aligned}
$$

For the convenience of the reader, we will first give a brief summary of the pre-commitment and time consistent policies.

\subsection{Pre-commitment Policy}

We review here the pre-commitment policy, as discussed in [29]. In this case, the optimal policy solves the following optimization problem,

$$
\mathcal{V}(w, t)=\sup _{p(s \geq t, W(s))}\left\{E_{t, w}^{p}[W(T)]-\lambda \operatorname{Var}_{t, w}^{p}[W(T)] \mid W(t)=w\right\},
$$

where $W(T), t<T$ is the investor's terminal wealth, subject to stochastic process $(2.2)$, and where $\lambda>0$ is a given Lagrange multiplier. The multiplier $\lambda$ can be interpreted as a coefficient of risk aversion. The optimal policy for (2.4) is called a pre-commitment policy [5]. 
Let $p_{t}^{*}(s, W(s)), s \geq t$, be the optimal policy for problem (2.4). Then, $p_{t+\Delta t}^{*}(s, W(s)), s \geq t+\Delta t$, is the optimal policy for

$$
\mathcal{V}(w, t+\Delta t)=\sup _{p(s \geq t+\Delta t, W(s))}\left\{E_{t+\Delta t, w}^{p}[W(T)]-\lambda V a r_{t+\Delta t, w}^{p}[W(T)] \mid W(t+\Delta t)=w\right\} .
$$

However, in general

$$
p_{t}^{*}(s, W(s)) \neq p_{t+\Delta t}^{*}(s, W(s)) ; s \geq t+\Delta t,
$$

i.e. solution of problem (2.4) is not time-consistent. Therefore, a dynamic programming principle cannot be directly applied to solve this problem. However, problem (2.4) can be embedded into a class of auxiliary stochastic Linear-Quadratic (LQ) problems using the method in [32, 21]. Alternatively, equation (2.4) can be posed as a convex optimization problem [22, 6, 1, 17]. More precisely, if $p_{t}^{*}(s, W(s))$ is the optimal control of equation (2.4), then there exists a $\gamma(t, w)$, such that $p_{t}^{*}(s, W(s))$ is also the optimal control of

$$
\inf _{p(s \geq t, W(s))}\left\{E_{t, w}^{p}\left[\left(W(T)-\frac{\gamma(t, w)}{2}\right)^{2}\right] \mid W(t)=w\right\} .
$$

Hence we can solve for $p_{t}^{*}(s, W(s))$ using dynamic programming. Note that this does not contradict our assertion that the optimal policy for equation (2.4) is not time consistent, since in general $\gamma(t, w)$ depends on the initial point $(t, w)$ (see Remark 1.1 in [7, and [19]). Problem 2.7 can be determined from the solution of an Hamilton Jacobi Bellman (HJB) equation. We have discussed the numerical solution of the resulting HJB equation in detail in [29].

\subsection{Time-consistent Policy}

In [30, we focused on the so called time-consistent policy. We can determine the time-consistent policy by solving problem (2.4) with an additional constraint,

$$
p_{t}^{*}(s, W(s))=p_{t^{\prime}}^{*}(s, W(s)) ; s \geq t^{\prime}, t^{\prime} \in[t, T] .
$$

In other words, we optimize problem (2.4), given that we follow the optimal policy in the future, which is determined by solving (2.4) at each future instant. Obviously, dynamic programming can be applied to the time-consistent problem. We have discussed the numerical algorithm for determining the optimal time-consistent policy in [30].

Remark 2.1 We follow the definition of a time consistent policy as given in [5], with a constant risk aversion parameter. Note that in [8], it is suggested that a wealth dependent risk aversion parameter is more meaningful. Some computations with a wealth dependent risk aversion parameter are given in [30]. However, we will use the original form with a constant risk aversion, in the following. See Remark 2.2.

\subsection{Mean Quadratic Variation}

Instead of using the variance/standard deviation as the risk measure, we can use the quadratic variation [9], $\int_{t}^{T}\left(d W_{s}\right)^{2}$. From equation 2.2 we have

$$
\left(d W_{t}\right)^{2}=(p(t, W(t)) \sigma W(t))^{2} d t,
$$


and consequently, we obtain,

$$
\int_{t}^{T}\left(d W_{s}\right)^{2}=\int_{t}^{T}(\sigma p(s, W(s)) W(s))^{2} d s
$$

Remark 2.2 (Relation to Time Consistent Mean Variance) In [7], the following rather surprising result is obtained. Without constraints (the allowing bankruptcy case, discussed in later sections), if $\int_{t}^{T}\left(e^{r(T-s)} d W_{s}\right)^{2}$ is used as the risk measure, the mean quadratic variation strategy has the same solution as the time-consistent strategy. The term $\left(e^{r(T-s)} d W_{s}\right)^{2}$ represents the future value of the instantaneous risk due to investing $p W$ (in monetary amount) in the risky asset. In order to facilitate comparison with various alternative strategies, we will use the risk measure

$$
\int_{t}^{T}\left(e^{r(T-s)} d W_{s}\right)^{2}=\int_{t}^{T}\left(e^{r(T-s)} \sigma p(s, W(s)) W(s)\right)^{2} d s
$$

in the following. Note that as discussed in [16], risk measure [2.11) is commonly used in optimal trade execution (with $r=0$ ).

Using equation (2.11) as a risk measure, we seek the optimal policy which solves the following optimization problem,

$$
\mathcal{V}(w, t)=\sup _{p(s \geq t, W(s))} E_{t, w}^{p}\left\{W(T)-\lambda \int_{t}^{T}\left(e^{r(T-s)} \sigma p(s, W(s)) W(s)\right)^{2} d s \mid W(t)=w\right\},
$$

where $\lambda$ is a given Lagrange multiplier, subject to stochastic process $(2.2)$. Let $p_{t}^{*}(s, W(s)), s \geq t$, be the optimal policy for problem (2.12). Then clearly,

$$
p_{t}^{*}(s, W(s))=p_{t^{\prime}}^{*}(s, W(s)) ; s \geq t^{\prime}, t^{\prime} \in[t, T] .
$$

Hence, dynamic programming can be directly applied to this problem.

\section{Mean Quadratic Variation Wealth Case}

In this section, we formulate the mathematical model for the optimal mean quadratic variation investment strategy. Let,

$$
\begin{aligned}
& \mathbb{D}:=\text { the set of all admissible wealth } W(t) \text {, for } 0 \leq t \leq T \\
& \mathbb{P}:=\text { the set of all admissible controls } p(t, W(t)) \text {, for } 0 \leq t \leq T \text { and } W(t) \in \mathbb{D} .
\end{aligned}
$$

Remark 3.1 Strictly speaking, we choose $\mathbb{P}$ so as to enforce $W \in \mathbb{D}$. However, when the control problem is formulated as the solution to an $H J B P D E$, then we choose $\mathbb{D}$, and select a boundary condition which specifies a control which ensures that $W \in \mathbb{D}$.

We seek the solution of the optimization problem (2.12). Define

$$
\mathcal{V}(w, t)=\sup _{p \in \mathbb{P}} E_{t, w}^{p}\left\{W(T)-\lambda \int_{t}^{T}\left(e^{r(T-s)} \sigma p(s, W(s)) W(s)\right)^{2} d s \quad \mid W(t)=w\right\} .
$$


Let $\tau=T-t$. Then using equation (2.2) and Ito's Lemma, we have that $V(w, \tau)=\mathcal{V}(w, t)$ satisfies the HJB equation

$$
V_{\tau}=\sup _{p \in \mathbb{P}}\left\{\mu_{w}^{p} V_{w}+\frac{1}{2}\left(\sigma_{w}^{p}\right)^{2} V_{w w}-\lambda\left(e^{r \tau} \sigma p w\right)^{2}\right\} ; w \in \mathbb{D},
$$

with terminal condition

$$
V(w, 0)=w,
$$

and where

$$
\begin{aligned}
\mu_{w}^{p} & =\pi+w(r+p \sigma \xi) \\
\left(\sigma_{w}^{p}\right)^{2} & =(p \sigma w)^{2} .
\end{aligned}
$$

Since PDE (3.3) can be degenerate depending on the control, we have no reason to believe that the solution is smooth. For this reason, we seek the viscosity solution to equation (3.3). This is discussed further in Section 5.1 .

In order to trace out the efficient frontier solution (in terms of mean and quadratic variation of the wealth) of problem (2.12), we proceed in the following way. Pick an arbitrary value of $\lambda$ and solve problem (2.12), which determines the optimal control $p^{*}(t, w)$. We also need to determine $E_{t=0, w}^{p^{*}}[W(T)]$.

Let $G=G(w, \tau)=E\left[W(T) \mid W(t)=w, p(s \geq t, w)=p^{*}(s \geq t, w)\right]$. Then $G$ is given from the solution to

$$
G_{\tau}=\left\{\mu_{w}^{p} G_{w}+\frac{1}{2}\left(\sigma_{w}^{p}\right)^{2} G_{w w}\right\}_{p(T-\tau, w)=p^{*}(T-\tau, w)} \quad ; w \in \mathbb{D},
$$

with the terminal condition

$$
G(w, 0)=w .
$$

Since the most costly part of the solution of equation (3.3) is the determination of the optimal control $p^{*}$, solution of equation $(3.6)$ is very inexpensive, once $p^{*}$ is known.

Then, if

$$
\begin{aligned}
& V\left(\hat{w}_{0}, T\right)=E_{t=0, \hat{w}_{0}}^{p^{*}}\left\{W(T)-\lambda \int_{0}^{T}\left(e^{r(T-s)} \sigma p^{*}(s, W(s)) W(s)\right)^{2} d s \mid W(0)=\hat{w}_{0}\right\}, \\
& G\left(\hat{w}_{0}, T\right)=E_{t=0, \hat{w}_{0}}^{p^{*}}\left\{W(T) \mid W(0)=\hat{w}_{0}\right\},
\end{aligned}
$$

we have that

$$
\begin{aligned}
& E_{t=0, \hat{w}_{0}}^{p^{*}}\left\{\int_{0}^{T}\left(e^{r(T-s)} \sigma p^{*}(s, W(s)) W(s)\right)^{2} d s \mid W(0)=\hat{w}_{0}\right\} \\
& =\left(G\left(\hat{w}_{0}, T\right)-V\left(\hat{w}_{0}, T\right)\right) / \lambda .
\end{aligned}
$$

It is useful also to determine the variance of the terminal wealth, $\operatorname{Var}_{t=0, w}^{p^{*}}[W(T)]$, under the optimal strategy in terms of mean quadratic variation. Let $F=F(w, \tau)=E\left[W(T)^{2} \mid W(t)=\right.$ $\left.w, p(s \geq t, w)=p^{*}(s \geq t, w)\right]$. Then $F$ is given from the solution to

$$
F_{\tau}=\left\{\mu_{w}^{p} F_{w}+\frac{1}{2}\left(\sigma_{w}^{p}\right)^{2} F_{w w}\right\}_{p(T-\tau, w)=p^{*}(T-\tau, w)} ; w \in \mathbb{D},
$$


with the terminal condition

$$
F(w, 0)=w^{2} .
$$

Assuming $F\left(\hat{w}_{0}, T\right), G\left(\hat{w}_{0}, T\right)$ are known, for a given $\lambda$, we can then compute the pair

$$
\left(\operatorname{Var}_{t=0, \hat{w}_{0}}^{p^{*}}[W(T)], E_{t=0, \hat{w}_{0}}^{p^{*}}[W(T)]\right)
$$

from $\operatorname{Var}_{t=0, \hat{w}_{0}}^{p^{*}}[W(T)]=F\left(\hat{w}_{0}, T\right)-\left[G\left(\hat{w}_{0}, T\right)\right]^{2}$ and $E_{t=0, \hat{w}_{0}}^{p^{*}}[W(T)]=G\left(\hat{w}_{0}, T\right)$.

Remark 3.2 If we allow an unbounded control set $\mathbb{P}=(-\infty,+\infty)$, then the total wealth can become negative (i.e. bankruptcy is allowed). In this case $\mathbb{D}=(-\infty,+\infty)$. If the control set $\mathbb{P}$ is bounded, i.e. $\mathbb{P}=\left[p_{\min }, p_{\max }\right]$, then negative wealth is not possible, in which case $\mathbb{D}=[0,+\infty)$. We can also have $p_{\max } \rightarrow+\infty$, but prohibit negative wealth, in which case $\mathbb{D}=[0,+\infty)$ as well.

\subsection{Localization}

Let,

$$
\hat{\mathbb{D}}:=\text { a finite computational domain which approximates the set } \mathbb{D} \text {. }
$$

In order to solve the PDEs (3.3), (3.6) and (3.10), we need to use a finite computational domain, $\hat{\mathbb{D}}=\left[w_{\min }, w_{\max }\right]$. When $w \rightarrow \pm \infty$, we assume that

$$
\begin{aligned}
& V(w \rightarrow \pm \infty, \tau) \simeq H_{1}(\tau) w^{2} \\
& G(w \rightarrow \pm \infty, \tau) \simeq J_{1}(\tau) w \\
& F(w \rightarrow \pm \infty, \tau) \simeq I_{1}(\tau) w^{2}
\end{aligned}
$$

then, ignoring lower order terms and taking into account the initial conditions (3.4), (3.7), (3.11),

$$
\begin{aligned}
V(w \rightarrow \pm \infty, \tau) & \simeq \frac{\lambda e^{r \tau} k_{2}}{2 k_{1}+k_{2}}\left(1-e^{\left(2 k_{1}+k_{2}\right) \tau}\right) w^{2}, \\
G(w \rightarrow \pm \infty, \tau) & \simeq e^{k_{1} \tau} w \\
F(w \rightarrow \pm \infty, \tau) & \simeq e^{\left(2 k_{1}+k_{2}\right) \tau} w^{2}
\end{aligned}
$$

where $k_{1}=r+p \sigma \xi$ and $k_{2}=(p \sigma)^{2}$. We consider three cases.

\subsubsection{Allowing Bankruptcy, Unbounded Controls}

In this case, we assume there are no constraints on $W(t)$ or on the control $p$, i.e., $\mathbb{D}=(-\infty,+\infty)$ and $\mathbb{P}=(-\infty,+\infty)$. Since $W(t)=w$ can be negative, bankruptcy is allowed. We call this case the allowing bankruptcy case.

Our numerical problem uses

$$
\hat{\mathbb{D}}=\left[w_{\min }, w_{\max }\right],
$$

where $\hat{\mathbb{D}}=\left[w_{\min }, w_{\max }\right]$ is an approximation to the original set $\mathbb{D}=(-\infty,+\infty)$.

Applying equation $(3.13)$ at finite $\left[w_{\min }, w_{\max }\right]$ will cause some error. However, we can make these errors small by choosing large values for $\left(\left|w_{\min }\right|, w_{\max }\right)$. We have verified this in [29, 30], and numerical tests show that this property holds for the mean quadratic variation strategy as well. If asymptotic forms of the solution are unavailable, we can use any reasonable estimate for $p^{*}$ for $|w|$ large, and the error will be small if $\left(\left|w_{\min }\right|, w_{\max }\right)$ are sufficiently large [3]. 


\subsubsection{No Bankruptcy, No Short Sales}

In this case, we assume that bankruptcy is prohibited and the investor cannot short the stock index, i.e., $\mathbb{D}=[0,+\infty)$ and $\mathbb{P}=[0,+\infty)$. We call this case the no bankruptcy (or bankruptcy prohibition) case.

Our numerical problem uses,

$$
\hat{\mathbb{D}}=\left[0, w_{\max }\right] .
$$

The boundary conditions for $V, G, F$ at $w=w_{\max }$ are given by equations (3.14). We prohibit the possibility of bankruptcy $(W(t)<0)$ by requiring that (see Remark 3.3$) \lim _{w \rightarrow 0}(p w)=0$, so that equations (3.3), (3.6) and (3.10) reduce to (at $w=0)$

$$
\begin{aligned}
V_{\tau}(0, \tau) & =\pi V_{w}, \\
G_{\tau}(0, \tau) & =\pi G_{w}, \\
F_{\tau}(0, \tau) & =\pi F_{w} .
\end{aligned}
$$

Another way of deriving this boundary condition is to note that we can rewrite equation (3.3) using the control $q=p w$. In this case $q$ is the dollar amount invested in the risky asset. We can prohibit negative wealth by requiring that the amount invested in the risky asset $q=0$ at $w=0$.

Remark 3.3 It is important to know the behavior of $p^{*} w$ as $w \rightarrow 0$, since it helps us determine whether negative wealth is admissible or not. As shown above, negative wealth is admissible for the case of allowing bankruptcy. In the case of no bankruptcy, although $p \in \mathbb{P}=[0,+\infty)$, we must have $\lim _{w \rightarrow 0}(p w)=0$ so that $W(t) \geq 0$ for all $0 \leq t \leq T$. In particular, we need to make sure that the optimal strategy never generates negative wealth, i.e., Probability $\left(W(t)<0 \mid p^{*}\right)=0$ for all $0 \leq t \leq T$. We will see from the numerical solutions that boundary condition (3.17) does in fact result in $\lim _{w \rightarrow 0}\left(p^{*} w\right)=0$. Hence, negative wealth is not admissible under the optimal strategy. More discussion of this issue are given in Section 6 .

Remark 3.4 (Behavior of $W(t), W \rightarrow 0$ ) The precise behavior of the controlled stochastic process will depend on the behavior of $p w$ as $w \rightarrow 0$, from the solution of the HJB equation (3.3). If $p w \rightarrow C_{1} w^{\gamma}, w \rightarrow 0, C_{1}$ independent of $w$, then the behaviour of $W(t)$ near $W=0$ can be determined from the usual Feller conditions. Equivalently, and more germane for PDE analysis, we can examine the Fichera function [15] to determine if boundary conditions are required at $w=0$. We have the following possibilities

- $\gamma \geq 1, w=0$ is unattainable, and no boundary condition is required;

- $1 / 2<\gamma<1$, which implies that $w=0$ is attainable but no boundary condition is required;

- $\gamma=1 / 2, w=0$ is attainable, and no boundary condition is required if $\pi \geq C_{1}^{2} \sigma^{2} / 2$, otherwise a boundary condition is required;

- $0<\gamma<1 / 2, w=0$ is attainable, and we need to supply a boundary condition. In this case we apply a reflecting condition (from equation (3.17)) if $\pi>0$ and an absorbing condition if $\pi=0$. 
Note that for numerical purposes, we always apply conditions (3.17). In some cases, as noted above, boundary conditions at $w=0$ are not actually required, but this does not cause any problems if the boundary condition is superfluous [26, 15]. This is convenient, since of course we do not know the precise behavior of $(p w), w \rightarrow 0$ until we solve the HJB equation (3.3) and determine the control $p$.

Remark 3.5 (Economic Interpretation of the Conditions on pw) The quantity pw is the dollar amount invested in the risky asset. Consequently, the above conditions on pw can be interpreted in economic terms. For example, we prohibit negative wealth by requiring that the dollar amount invested in the risky asset must tend to zero as the the investor's wealth tends to zero. Note that this economically reasonable condition permits $p$ to be finite or infinite at $w=0$.

\subsubsection{No Bankruptcy, Bounded Control}

This is a realistic case, in which we assume that bankruptcy is prohibited and infinite borrowing is not allowed. As a result, $\mathbb{D}=[0,+\infty)$ and $\mathbb{P}=\left[0, p_{\max }\right]$. We call this case the bounded control case. For example, typical margin requirements on a brokerage account limit borrowing to $50 \%$ of the market value of the assets in the account. This would translate into a value of $p_{\max }=1.5$.

Our numerical problem uses,

$$
\hat{\mathbb{D}}=\left[0, w_{\max }\right],
$$

where $w_{\max }$ is an approximation to the infinity boundary. Other assumptions and the boundary conditions for $V$ and $G$ are the same as those of no bankruptcy case introduced in Section 3.1.2. Note that for the bounded control case, the control is finite, thus $\lim _{w \rightarrow 0}(p w)=0$ and negative wealth is not admissible.

We summarize the various cases in Table 1

\begin{tabular}{|c|c|c|}
\hline Case & $\hat{\mathbb{D}}$ & $\mathbb{P}$ \\
\hline Bankruptcy & {$\left[w_{\min }, w_{\max }\right]$} & $(-\infty,+\infty)$ \\
No Bankruptcy & {$\left[0, w_{\max }\right]$} & {$[0,+\infty)$} \\
Bounded Control & {$\left[0, w_{\max }\right]$} & {$\left[0, p_{\max }\right]$} \\
\hline
\end{tabular}

TABLE 1: Summary of cases.

\subsection{Special Case: Reduction to the Classic Multi-period Portfolio Selection Problem}

The classic multi-period portfolio selection problem can be stated as the following: given some investment choices (assets) in the market, an investor seeks an optimal asset allocation strategy over a period $T$ with an initial wealth $\hat{w}_{0}$. This problem has been widely studied [24, 32, 21, 23, 6, 22, . If we use the mean variance approach to solve this problem, then the best strategy $p^{*}(w, t)$ can be defined as a solution of problem (2.4). We still assume there is one risk free bond and one risky asset in the market. In this case,

$$
\begin{aligned}
d W & =(r+p \xi \sigma) W d t+p \sigma W d Z_{1} \\
W(t=0) & =\hat{w}_{0}>0 .
\end{aligned}
$$


Clearly, the pension plan problem we introduced previously can be reduced to the classic multiperiod portfolio selection problem by simply setting the contribution rate $\pi=0$. All equations and boundary conditions stay the same.

\section{Wealth-to-income Ratio Case}

In the previous section, we considered the expected value and variance/quadratic variation of the terminal wealth in order to construct an efficient frontier. In [10, it is argued that at retirement, a pension plan member will be concerned with preservation of her standard of living. This suggests measuring wealth in terms of a numeraire computed based on the investor's pre-retirement salary. This approach for retirement saving takes into account the stochastic feature of the plan member's lifetime salary progression, as well as the stochastic nature of the investment assets.

In this section, instead of the terminal wealth, we determine the mean variance efficient strategy in terms of the terminal wealth-to-income ratio $X=\frac{W}{Y}$, where $Y$ is the annual salary in the year before she retires. As noted in [10], this is consistent with the habit formation model developed in [28, 12 .

In the following, we give a brief overview of the model developed in [10]. We still assume there are two underlying assets in the pension plan: one is risk free and the other is risky. Recall from equation (2.1) that the risky asset $S$ follows the Geometric Brownian Motion,

$$
d S=(r+\xi \sigma) S d t+\sigma S d Z_{1} .
$$

Suppose that the plan member continuously pays into the pension plan at a fraction $\pi$ of her yearly salary $Y$, which follows the process

$$
d Y=\left(r+\mu_{Y}\right) Y d t+\sigma_{Y_{0}} Y d Z_{0}+\sigma_{Y_{1}} Y d Z_{1}
$$

where $\mu_{Y}, \sigma_{Y_{0}}$ and $\sigma_{Y_{1}}$ are constants, and $d Z_{0}$ is another increment of a Wiener process, which is independent of $d Z_{1}$. Let $p$ denote the proportion of this wealth invested in the risky asset $S$, and let $1-p$ denote the fraction of wealth invested in the risk-free asset. Then

$$
\begin{aligned}
d W & =(r+p \xi \sigma) W d t+p \sigma W d Z_{1}+\pi Y d t \\
W(t=0) & =\hat{w}_{0} \geq 0 .
\end{aligned}
$$

Define a new state variable $X(t)=W(t) / Y(t)$, then by Ito's Lemma, we obtain

$$
\begin{aligned}
d X= & {\left[\pi+X\left(-\mu_{Y}+p \sigma\left(\xi-\sigma_{Y_{1}}\right)+\sigma_{Y_{0}}^{2}+\sigma_{Y_{1}}^{2}\right)\right] d t } \\
& -\sigma_{Y_{0}} X d Z_{0}+X\left(p \sigma-\sigma_{Y_{1}}\right) d Z_{1} \\
X(t=0)= & \hat{x}_{0} \geq 0 .
\end{aligned}
$$

Let

$$
\begin{aligned}
\mu_{X}^{p} & =\pi+X\left(-\mu_{Y}+p \sigma\left(\xi-\sigma_{Y_{1}}\right)+\sigma_{Y_{0}}^{2}+\sigma_{Y_{1}}^{2}\right) \\
\left(\sigma_{X}^{p}\right)^{2} & =\left(\sigma_{X}^{p}(p(t, X(t)), X(t))^{2}\right. \\
& =X^{2}\left(\sigma_{Y_{0}}^{2}+\left(p \sigma-\sigma_{Y_{1}}\right)^{2}\right)
\end{aligned}
$$


then the control problem is to determine the control $p(t, X(t)=x)$ such that $p(t, x)$ maximizes

$$
\mathcal{V}(x, t)=\sup _{p \in \mathbb{P}} E_{t, x}^{p}\left\{X(T)-\lambda \int_{t}^{T} e^{2 r^{\prime}(T-s)}\left(\sigma_{X}^{p}\right)^{2} d s \mid X(t)=x\right\}
$$

subject to stochastic process 4.4, where $r^{\prime}=-\mu_{Y}+\sigma_{Y_{0}}^{2}+\sigma_{Y_{1}}^{2}$. Note that we have posed the problem in terms of the future value of the quadratic variation using $r^{\prime}$ as the discount factor. For the wealth case, with no constraints on the controls, the analytic solution for the time-consistent mean variance policy is identical to the mean quadratic strategy (2.12) [7. However, there does not appear to be an analytic solution available for the wealth-to-income ratio case, hence we use $r^{\prime}$ as the effective drift rate (when there is no investment in the risky asset). There are clearly other possibilities here.

Let $\tau=T-t$. Then $V(x, \tau)=\mathcal{V}(x, t)$ satisfies the HJB equation

$$
V_{\tau}=\sup _{p \in \mathbb{P}}\left\{\mu_{x}^{p} V_{x}+\frac{1}{2}\left(\sigma_{x}^{p}\right)^{2} V_{x x}-\lambda e^{2 r^{\prime} \tau}\left(\sigma_{x}^{p}\right)^{2}\right\} ; x \in \mathbb{D},
$$

with terminal condition

$$
V(x, 0)=x .
$$

We still use $\mathbb{D}$ and $\mathbb{P}$ as the sets of all admissible wealth-to-income ratio and control. As before, we let $\hat{\mathbb{D}}$ be the localized computational domain.

We also solve for $G(x, \tau)=E\left[X(T) \mid X(t)=x, p(s \geq t, x)=p^{*}(s \geq t, x)\right]$ and $F(x, \tau)=$ $E\left[X(T)^{2} \mid X(t)=x, p(s \geq t, x)=p^{*}(s \geq t, x)\right]$ using

$$
\begin{aligned}
& G_{\tau}=\left\{\mu_{x}^{p} G_{x}+\frac{1}{2}\left(\sigma_{x}^{p}\right)^{2} G_{x x}\right\}_{p(T-\tau, x)=p^{*}(T-\tau, x)} ; x \in \mathbb{D}, \\
& F_{\tau}=\left\{\mu_{x}^{p} F_{x}+\frac{1}{2}\left(\sigma_{x}^{p}\right)^{2} F_{x x}\right\}_{p(T-\tau, x)=p^{*}(T-\tau, x)} ; x \in \mathbb{D},
\end{aligned}
$$

with terminal condition

$$
\begin{aligned}
G(x, 0) & =x . \\
F(x, 0) & =x^{2} .
\end{aligned}
$$

We can then use the method described in Section 3 to trace out the efficient frontier solution of problem 4.6).

We consider the cases: allowing bankruptcy $(\mathbb{D}=(-\infty,+\infty), \mathbb{P}=(-\infty,+\infty))$, no bankruptcy $(\mathbb{D}=[0,+\infty), \mathbb{P}=[0,+\infty))$, and bounded control $\left(\mathbb{D}=[0,+\infty), \mathbb{P}=\left[0, p_{\max }\right]\right)$. For computational purposes, we localize the problem to to $\hat{\mathbb{D}}=\left[x_{\min }, x_{\max }\right]$, and apply boundary conditions as in Section 3.1. More precisely, if $x=0$ is a boundary, with $X<0$ prohibited, then $\lim _{w \rightarrow 0}(p x)=0$, and hence

$$
\begin{aligned}
V_{\tau}(0, \tau) & =\pi V_{x}, \\
G_{\tau}(0, \tau) & =\pi G_{x}, \\
F_{\tau}(0, \tau) & =\pi F_{x} .
\end{aligned}
$$

The boundary conditions at $x \rightarrow \pm \infty$ are given in equation (3.14), but using $x$ instead of $w$ and $r^{\prime}$ instead of $r$ with $k_{1}=-\mu_{Y}+p \sigma\left(\xi-\sigma_{Y_{1}}\right)+\sigma_{Y_{0}}^{2}+\sigma_{Y_{1}}^{2}$ and $k_{2}=\sigma_{Y_{0}}^{2}+\left(p \sigma-\sigma_{Y_{1}}\right)^{2}$. 


\section{Discretization of the HJB PDE}

The numerical scheme to solve the PDEs is similar to the scheme used in [29]. We briefly describe the discretization scheme in this section, and refer readers to [29] for details.

Define,

$$
\mathcal{L}^{p} V \equiv a(z, p) V_{z z}+b(z, p) V_{z},
$$

where

$$
z=w \quad ; \quad a(z, p)=\frac{1}{2}\left(\sigma_{w}^{p}\right)^{2} ; b(z, p)=\mu_{w}^{p} ; d(z, p, \tau)=-\lambda\left(e^{r \tau} \sigma p w\right)^{2}
$$

(see equation (3.5)) for the wealth case introduced in Section 2 and

$$
z=x \quad ; \quad a(z, p)=\frac{1}{2}\left(\sigma_{x}^{p}\right)^{2} \quad ; \quad b(z, p)=\mu_{x}^{p} ; d(z, p, \tau)=-\lambda e^{2 r^{\prime} \tau}\left(\sigma_{x}^{p}\right)^{2}
$$

(see equation (4.5) for the wealth-to-income ratio case introduced in Section 4 . Then,

$$
V_{\tau}=\sup _{p \in \mathbb{P}}\left\{\mathcal{L}^{p} V+d(z, p, \tau)\right\}
$$

and

$$
\begin{aligned}
G_{\tau} & =\left\{\mathcal{L}^{p} G\right\}_{p=p^{*}}, \\
F_{\tau} & =\left\{\mathcal{L}^{p} F\right\}_{p=p^{*}} .
\end{aligned}
$$

Define a grid $\left\{z_{0}, z_{1}, \ldots, z_{q}\right\}$ with $z_{0}=z_{\min }, z_{q}=z_{\max }$ and let $V_{i}^{n}$ be a discrete approximation to $V\left(z_{i}, \tau^{n}\right)$. Set $P^{n}=\left[p_{0}^{n}, p_{1}^{n}, \ldots, p_{q}^{n}\right]^{\prime}$, with each $p_{i}^{n}$ a local optimal control at $\left(z_{i}, \tau^{n}\right)$. Let $P^{*}=\left\{P^{0}, P^{1}, \ldots, P^{N}\right\}$, where $\tau^{N}=T$. In other words, $P^{*}$ contains the discrete optimal controls for all $(i, n)$. Let $V^{n}=\left[V_{0}^{n}, \ldots, V_{q}^{n}\right]^{\prime}$, and let $\left(\mathcal{L}_{h}^{P^{n}} V^{n}\right)_{i}$ denote the discrete form of the differential operator (5.1) at node $\left(z_{i}, \tau^{n}\right)$. The operator (5.1) can be discretized using forward, backward or central differencing in the $z$ direction to give

$$
\left(\mathcal{L}_{h}^{P^{n+1}} V^{n+1}\right)_{i}=\alpha_{i}^{n+1} V_{i-1}^{n+1}+\beta_{i}^{n+1} V_{i+1}^{n+1}-\left(\alpha_{i}^{n+1}+\beta_{i}^{n+1}\right) V_{i}^{n+1} .
$$

Here $\alpha_{i}, \beta_{i}$ are defined in Appendix A.

Equation (5.4) can now be discretized using fully implicit timestepping along with the discretization (5.7) to give

$$
\frac{V_{i}^{n+1}-V_{i}^{n}}{\Delta \tau}=\sup _{P^{n+1} \in \hat{P}}\left\{\left(\mathcal{L}_{h}^{P^{n+1}} V^{n+1}\right)_{i}+d\left(z_{i},\left[P^{n+1}\right]_{i}, \tau\right)\right\}
$$

where $\hat{P}=\left\{\left[p_{0}, p_{1}, \ldots, p_{q}\right]^{\prime} \mid p_{i} \in \mathbb{P}, 0 \leq i \leq q\right\}$. With $P^{n+1}$ given from equation (5.8), then equations (5.5) and (5.6) can be discretized as

$$
\begin{aligned}
& \frac{G_{i}^{n+1}-G_{i}^{n}}{\Delta \tau}=\left\{\left(\mathcal{L}_{h}^{P^{n+1}} G^{n+1}\right)_{i}\right\}, \\
& \frac{F_{i}^{n+1}-F_{i}^{n}}{\Delta \tau}=\left\{\left(\mathcal{L}_{h}^{P^{n+1}} F^{n+1}\right)_{i}\right\} .
\end{aligned}
$$


Note that $\alpha_{i}^{n+1}=\alpha_{i}^{n+1}\left(p_{i}^{n+1}\right)$ and $\beta_{i}^{n+1}=\beta_{i}^{n+1}\left(p_{i}^{n+1}\right)$, that is, the discrete equation coefficients are functions of the local optimal control $p_{i}^{n+1}$. This makes equations (5.8) highly nonlinear in general. We use a policy type iteration [29] to solve the non-linear discretized algebraic equation (5.8).

Given an initial value $\hat{z}_{0}$, we can use the algorithm introduced in [29] to obtain the efficient frontier.

\subsection{Convergence to the Viscosity Solution}

PDEs (5.5) and (5.6) are linear, since the optimal control is pre-computed. We can then obtain classical solutions of the linear PDEs (5.5) and (5.6). However, PDE (5.4) is highly nonlinear, so the classical solution may not exist in general. In this case, we are seeking the viscosity solution [2, 13 .

In [27], examples were given in which seemingly reasonable discretizations of nonlinear HJB PDEs were unstable or converged to the incorrect solution. It is important to ensure that we can generate discretizations which are guaranteed to converge to the viscosity solution [2, 13. In the case of bounded controls, on a finite computational domain, the PDE (5.4) satisfies the conditions required in [4], so that a strong comparison property holds.

In the case of allowing bankruptcy and no bankruptcy (see Table 1), the control $p$ is unbounded near $w=0$, which would violate some of the conditions required in 44. However, we can avoid these difficulties, if we rewrite PDE (5.4) in terms of the control $q=p w$. From the analytic solutions, we note that $q$ is bounded on a finite computational domain, hence a strong comparison property holds in this case as well. In fact, our numerical implementation for these two cases does in fact use $q=p w$ as the control, as discussed in [30].

Following the same proof given in [29], we can show that scheme (5.8) converges to the viscosity solution of equation (5.4), assuming that (5.4) satisfies a strong comparison principle. We refer readers to [29] for details.

\section{Numerical Results: Mean Quadratic Variation}

In this section we examine the numerical results for the strategy of minimizing the quadratic variation. We consider two risk measures when we construct efficient frontiers. One measure is the usual standard deviation, and the other measure is the expected future value of the quadratic variation, $E\left[\int_{0}^{T}\left(e^{r(T-s)} d W_{s}\right)^{2}\right]$. We use the notation $Q_{-} s t d_{t=0, w}^{p^{*}}[W(T)]$ to denote

$$
Q_{-} s t d_{t=0, w}^{p^{*}}[W(T)]=\left(E_{t=0, w}^{p^{*}}\left\{\int_{0}^{T}\left(e^{r(T-s)} \sigma p^{*}(s, W(s)) W(s)\right)^{2} d s \mid W(0)=w\right\}\right)^{1 / 2} .
$$

\subsection{Wealth Case}

When bankruptcy is allowed, as pointed out in [7], the mean quadratic variation strategy has the same solution as the time-consistent strategy. The analytic solutions for the time-consistent strategy are given in Section 7. Given the parameters in Table 2, if $\lambda=0.6$, the exact solution is $\left(\operatorname{Std}_{t=0, w}^{p^{*}}[W(T)], E_{t=0, w}^{p^{*}}[W(T)]\right)=(1.24226,6.41437)$. Table 3 and 4 show the numerical results. 
Table 3 reports the value of $V=E_{t=0, w}^{p^{*}}\left[W(T)-\lambda \int_{0}^{T}\left(e^{r(T-s)} d W_{s}\right)^{2}\right]$, which is the viscosity solution of the nonlinear HJB PDE (3.3). Table 3 shows that our numerical solution converges to the viscosity solution at a first order rate. Table 4 reports the value of $E_{t=0, w}^{p^{*}}[W(T)]$, which is the solution of the linear PDE (3.6). We also computed the values of $E_{t=0, w}^{p^{*}}\left[W(T)^{2}\right]$ (not shown in Tables), which is the the solution of PDE 3.10 . Given $E_{t=0, w}^{p^{*}}\left[W(T)^{2}\right]$ and $E_{t=0, w}^{p^{*}}[W(T)]$, the standard deviation can now be easily computed, which is also reported in Table 4. The results show that the numerical solutions of $\operatorname{Std}_{t=0, w}^{p^{*}}[W(T)]$ and $E_{t=0, w}^{p^{*}}[W(T)]$ converge to the analytic values at a first order rate as mesh and timestep size tends to zero.

\begin{tabular}{|c|c|c|c|}
\hline$r$ & 0.03 & $\xi$ & 0.33 \\
$\sigma$ & 0.15 & $\pi$ & 0.1 \\
$T$ & 20 years & $W(t=0)$ & 1 \\
\hline
\end{tabular}

TABle 2: Parameters used in the pension plan examples.

\begin{tabular}{|c|c|c|c|c|c|}
\hline $\begin{array}{c}\text { Nodes } \\
(\mathrm{W})\end{array}$ & Timesteps & $\begin{array}{c}\text { Nonlinear } \\
\text { iterations }\end{array}$ & $\begin{array}{c}\text { Normalized } \\
\text { CPU Time }\end{array}$ & $V(w=1, t=0)$ & Ratio \\
\hline 1456 & 320 & 640 & 1. & 5.49341 & \\
2912 & 640 & 1280 & 4.13 & 5.49092 & \\
5824 & 1280 & 2560 & 16.31 & 5.48968 & 2.008 \\
11648 & 2560 & 5120 & 66.23 & 5.48906 & 2.000 \\
23296 & 5120 & 10240 & 268.53 & 5.48875 & 2.000 \\
46592 & 10240 & 20480 & 1145.15 & 5.48860 & 2.067 \\
\hline
\end{tabular}

TABLE 3: Convergence study, wealth case, allowing bankruptcy. Fully implicit timestepping is applied, using constant timesteps. Parameters are given in Table 2, with $\lambda=0.6$. Values of $V=E_{t=0, w}^{p^{*}}\left[W(T)-\lambda \int_{0}^{T}\left(e^{r(T-t)} d w\right)^{2}\right]$ are reported at $(W=1, t=0)$. Ratio is the ratio of successive changes in the computed values for decreasing values of the discretization parameter $h$. CPU time is normalized. We take the CPU time used for the first test in this table as one unit of CPU time, which uses 1456 nodes for $W$ grid and 320 timesteps.

\begin{tabular}{|c|c|c|c|c|c|}
\hline $\begin{array}{c}\text { Nodes } \\
(W)\end{array}$ & Timesteps & $\operatorname{Std}_{t=0, w}^{p^{*}}[W(T)]$ & $E_{t=0, w}^{p^{*}}[W(T)]$ & $\begin{array}{c}\text { Ratio } \\
\text { for } \operatorname{Std}_{t=0, w}^{p^{*}}[W(T)]\end{array}$ & $\begin{array}{c}\text { Ratio } \\
\text { for } E[W(T)]\end{array}$ \\
\hline 1456 & 320 & 1.30652 & 6.41986 & 1.960 & \\
2912 & 640 & 1.27466 & 6.41711 & 1.972 & \\
5824 & 1280 & 1.25853 & 6.41574 & 1.975 & 2.007 \\
11648 & 2560 & 1.25041 & 6.41505 & 1.986 & 1.986 \\
23296 & 5120 & 1.24634 & 6.41471 & 1.995 & 2.029 \\
46592 & 10240 & 1.244300 & 6.41454 & 2.000 & 1.995098 \\
\hline
\end{tabular}

TABLE 4: Convergence study of the wealth case, allowing bankruptcy. Fully implicit timestepping is applied, using constant timesteps. The parameters are given in Table 2, with $\lambda=0.6$. Values of $S t d_{t=0, w}^{q^{*}}[W(T)]$ and $E_{t=0, w}^{q^{*}}[W(T)]$ are reported at $(W=1, t=0)$. Ratio is the ratio of successive changes in the computed values for decreasing values of the discretization parameter $h$. Analytic solution is $\left(S t d_{t=0, w}^{p^{*}}[W(T)], E_{t=0, w}^{p^{*}}[W(T)]\right)=(1.24226,6.41437)$. 
We also solve the problem for the no bankruptcy case and the bounded control case. The frontiers are shown in Figure 1, with parameters given in Table 2 and $(W=1, t=0)$. Figure 1 (a) shows the results obtained by using the standard deviation as the risk measure, and Figure 1 (b) shows the results obtained by using the quadratic variation as the risk measure. Note that, in both figures, the efficient frontiers pass through the same lowest point. At that point, the plan holder simply invests all her wealth in the risk free bond all the time, so the risk (standard deviation/quadratic variation) is zero. For both risk measures, the frontiers for the allowing bankruptcy case are straight lines. This result agrees with the results from the pre-commitment strategy [29] and the time-consistent strategy $[30$.

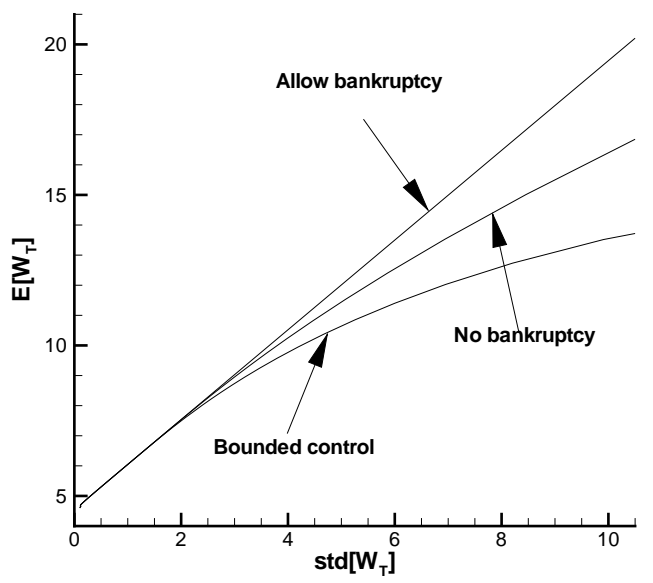

(a) Risk measure: std

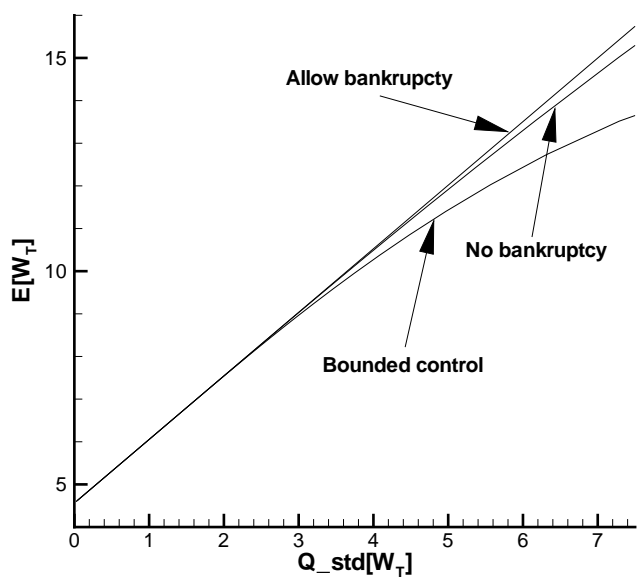

(b) Risk measure: $Q \_s t d$

FiguRE 1: Efficient frontiers (wealth case) for allowing bankruptcy $(\mathbb{D}=(-\infty,+\infty)$ and $\mathbb{P}=$ $(-\infty,+\infty))$, no bankruptcy $(\mathbb{D}=[0,+\infty)$ and $\mathbb{P}=[0,+\infty))$ and bounded control $(\mathbb{D}=[0,+\infty)$ and $\mathbb{P}=[0,1.5])$ cases. Parameters are given in Table 2. Values are reported at $(W=1, t=0)$. Figure (a) shows the frontiers with risk measure standard deviation. Figure (b) shows the frontiers with risk measure quadratic variation.

Figure 2 shows the effect of varying $\sigma$ while holding $\mu_{S}=r+\xi \sigma$ constant. In this case, the efficient frontiers are different values of $\sigma$ are well separated. Figure 3 shows the effect of varying $\sigma$ while holding $\xi$ constant. In this case, the curves for different values of $\sigma$ are much closer together. Note as well that if the value of $\sigma$ is increased with $\mu_{S}$ fixed, then the efficient frontier moves downward (Figure 2). On the other hand, as shown in Figure 3, the efficient frontier moves upward if $\sigma$ is increased with fixed $\xi$ (this makes the drift rate $\mu_{S}$ increase).

Figure 4 shows the values of the optimal control (the investment strategies) at different time $t$ for a fixed $T=20$. The parameters are given in Table 2, with bounded control $(p \in[0,1.5])$ and $\lambda=$ 0.604. Under these inputs, if $W(t=0)=1,\left(\operatorname{Std}_{t=0, w}^{p^{*}}[W(T)], E_{t=0, w}^{p^{*}}[W(T)]\right)=(1.23824,6.40227)$ and $Q \_s t d_{t=0, w}^{p^{*}}[W(T)]=1.52262$ from the finite difference solution. From this Figure, we can see that the control $p$ is an increasing function of time $t$ for a fixed $w$. This agrees with the results from the pre-commitment [29] and time-consistent strategies [30]. 


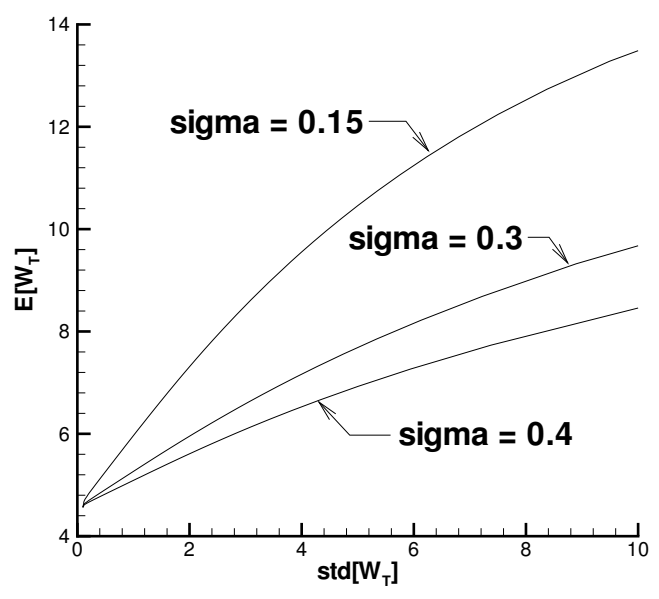

(a) Risk measure: std

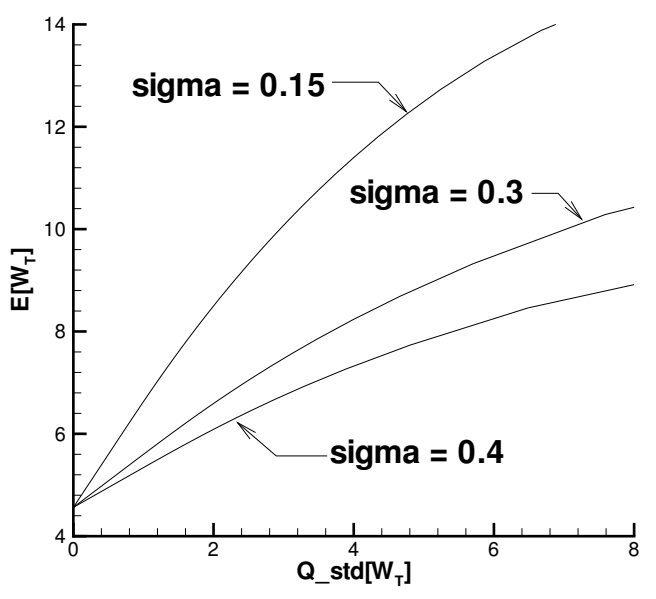

(b) Risk measure: Q_std

Figure 2: Efficient frontiers (wealth case), bounded control. We fix $\mu_{S}=r+\xi \sigma=.08$, and vary $\sigma$. Other parameters are given in Table 2. Values are reported at $(W=1, t=0)$. Figure (a) shows the frontiers with risk measure standard deviation. Figure (b) shows the frontiers with risk measure quadratic variation.

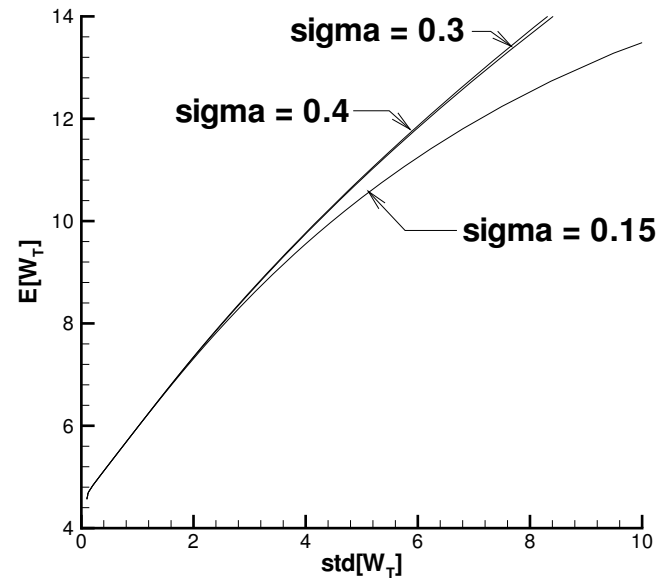

(a) Risk measure: std

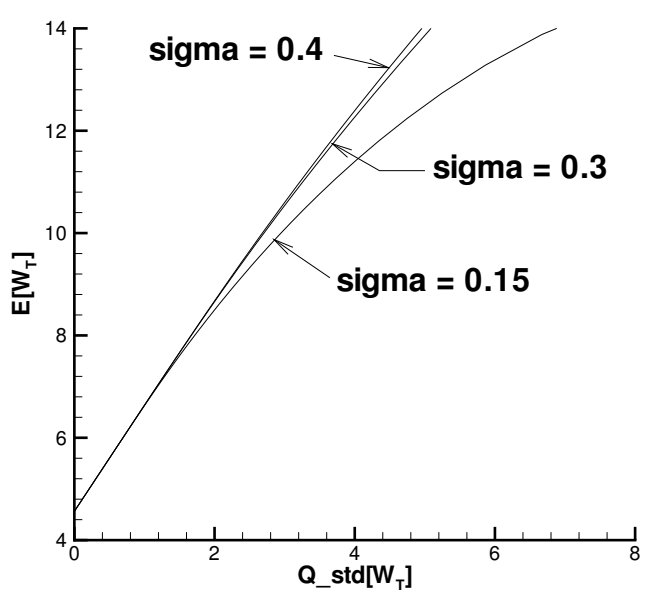

(b) Risk measure: $Q \_s t d$

FIGURE 3: Efficient frontiers (wealth case), bounded control. We fix $\xi=0.33$, and vary $\sigma$. Other parameters are given in Table 2. Values are reported at $(W=1, t=0)$. Figure (a) shows the frontiers with risk measure standard deviation. Figure (b) shows the frontiers with risk measure quadratic variation. 


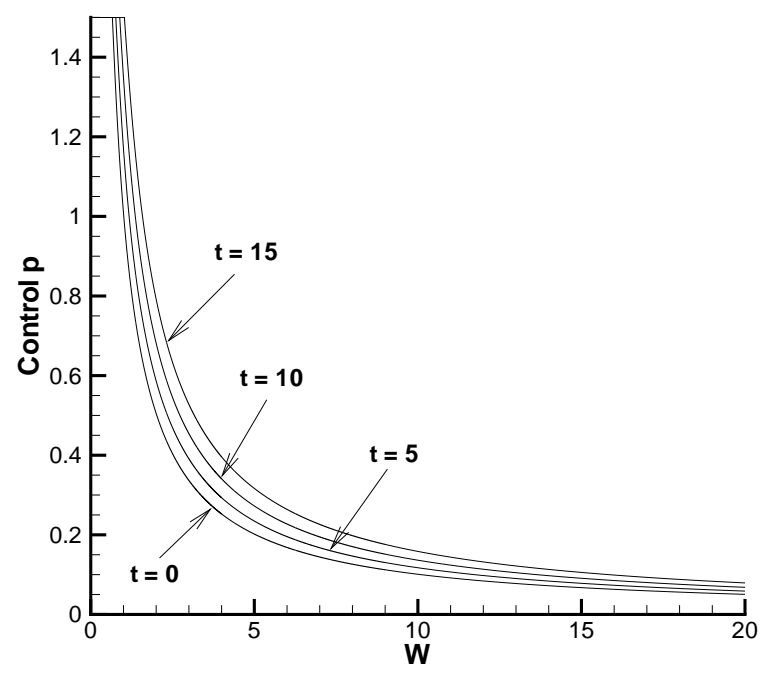

Figure 4: Optimal control as a function of $(W, t)$, bounded control case. Parameters are given in Table 2. with $\lambda=0.604$. Under these inputs, if $W(t=0)=1,\left(\operatorname{St}_{t=0, w}^{p^{*}}[W(T)], E_{t=0, w}^{p^{*}}[W(T)]\right)=$ $(1.23824,6.40227)$ and $Q \_s t d_{t=0, w}^{p^{*}}[W(T)]=1.52262$ from finite difference solution. Mean quadratic variation objective function.

Remark 6.1 As we discussed in Remark 3.3, in the case of bankruptcy prohibition, we have to have $\lim _{w \rightarrow 0}\left(p^{*} w\right)=0$ so that negative wealth is not admissible. Our numerical tests show that as $w$ goes to zero, $p^{*} w=O\left(w^{\gamma}\right)$. For a reasonable range of parameters, we have $0.9<\gamma<1$. Hence, this verifies that the boundary conditions (3.17) ensure that negative wealth is not admissible under the optimal strategy. This property also holds for the wealth-to-income ratio case.

\subsection{Multi-period Portfolio Selection}

As discussed in Section 3.2 , the wealth case can be reduced to the classic multi-period portfolio selection problem. Efficient frontier solutions of a particular multi-period portfolio selection problem are shown in Figure 5, with parameters in Table 2 but with $\pi=0$. Again, we consider three cases: allowing bankruptcy, no bankruptcy, and bounded control cases. Figure 5 (a) shows the results obtained by using the standard deviation as the risk measure, and Figure 5 (b) shows the results obtained by using the quadratic variation as the risk measure. As for the wealth case, in both figures, the frontiers for the allowing bankruptcy case are straight lines.

\subsection{Wealth-to-income Ratio Case}

In this section, we examine the wealth-to-income ratio case. Tables 6 and 7 show the numerical results for the bounded control case, using parameters in Table 5 . Table 6 reports the value of $V=E_{t=0, x}^{p^{*}}\left[X(T)-\lambda \int_{0}^{T}\left(e^{r(T-s)} d X_{s}\right)^{2}\right]$, which is the viscosity solution of nonlinear HJB PDE 4.7). Table 7 reports the value of $E_{t=0, x}^{p^{*}}[X(T)]$, which is the solution of the linear PDE $\sqrt[4.9]{ }$. We 


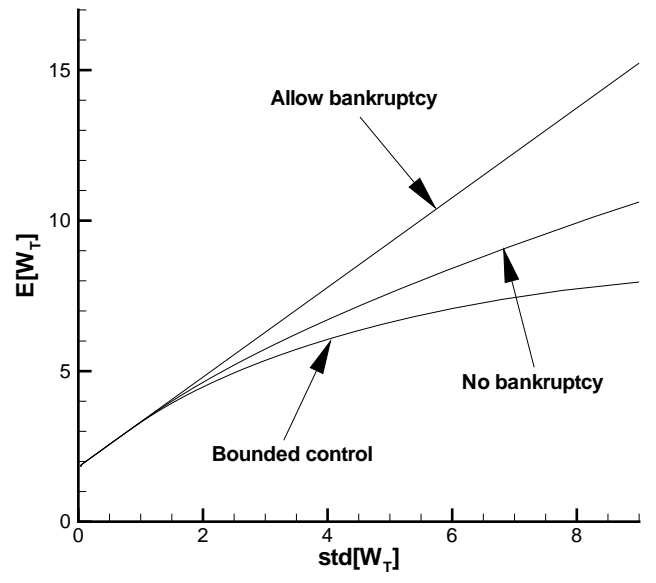

(a) Risk measure: std

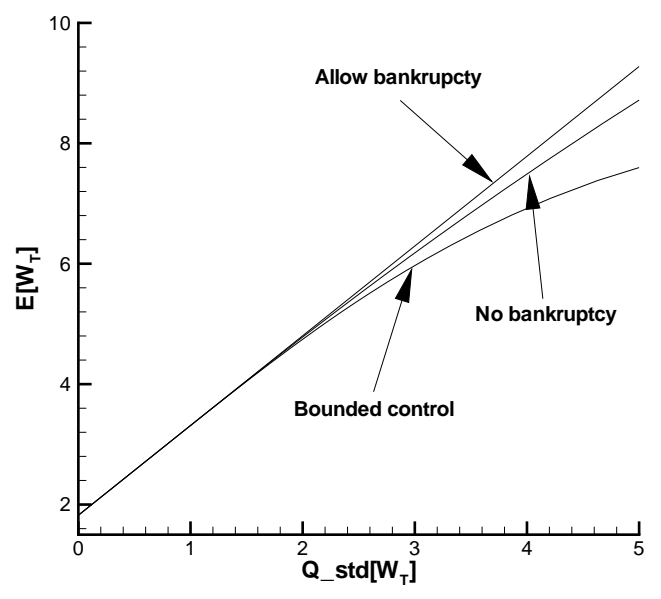

(b) Risk measure: Q_std

FIGURE 5: Efficient frontiers (multi-period portfolio selection) for allowing bankruptcy $(\mathbb{D}=$ $(-\infty,+\infty)$ and $\mathbb{P}=(-\infty,+\infty))$, no bankruptcy $(\mathbb{D}=[0,+\infty)$ and $\mathbb{P}=[0,+\infty)$ ) and bounded control $(\mathbb{D}=[0,+\infty)$ and $\mathbb{P}=[0,1.5])$ cases. Parameters are given in Table 2 but with $\pi=0$. Values are reported at $(W=1, t=0)$. Figure $(a)$ shows the frontiers with risk measure standard deviation. Figure (b) shows the frontiers with risk measure quadratic variation.

\begin{tabular}{|c|c|c|c|}
\hline$\mu_{y}$ & 0. & $\xi$ & 0.2 \\
$\sigma$ & 0.2 & $\sigma_{Y 1}$ & 0.05 \\
$\sigma_{Y 0}$ & 0.05 & $\pi$ & 0.1 \\
$T$ & 20 years & $\lambda$ & 0.25 \\
$\mathbb{Q}$ & {$[0,1.5]$} & $\mathbb{D}$ & {$[0,+\infty)$} \\
\hline
\end{tabular}

TABLE 5: Parameters used in the pension plan examples.

also computed the values of $E_{t=0, x}^{p^{*}}\left[X(T)^{2}\right]$ (not shown in tables), which is the the solution of PDE 4.10 .

Given $E_{t=0, x}^{p^{*}}\left[X(T)^{2}\right]$ and $E_{t=0, x}^{p^{*}}[X(T)]$, the standard deviation is easily computed. This is also reported in Table 7. The results show that the numerical solutions of $V$ and $E_{t=0, x}^{p^{*}}[X(T)]$ converges at a first order rate as mesh and timestep size tends to zero.

Efficient frontiers are shown in Figure 6, using parameters in Table 5 with $(X(0)=0.5 ; t=0)$. Figure 6 (a) shows the results obtained by using the standard deviation as the risk measure, and Figure 6 (b) shows the results obtained by using the quadratic variation as the risk measure. Note that, although the frontiers in both figures pass through the same lowest point, unlike the wealth case, the minimum standard deviation/quadratic variation for all strategies are no longer zero. Since the plan holder's salary is stochastic (equation (4.2) and the salary risk cannot be completely hedged away, there is no risk free strategy.

Figure 7 shows the values of the optimal control (the investment strategies) at different time $t$ for a fixed $T=20$. The parameters are given in Table 5, with $\lambda=0.2873$. Under these inputs, 


\begin{tabular}{|c|c|c|c|c|c|}
\hline $\begin{array}{c}\text { Nodes } \\
(\mathrm{W})\end{array}$ & Timesteps & $\begin{array}{c}\text { Nonlinear } \\
\text { iterations }\end{array}$ & $\begin{array}{c}\text { Normalized } \\
\text { CPU Time }\end{array}$ & $V(w=1, t=0)$ & Ratio \\
\hline 177 & 80 & 160 & 0.21 & 3.26653 & \\
353 & 160 & 320 & 1. & 3.26534 & \\
705 & 320 & 640 & 3.86 & 3.26476 & 2.052 \\
1409 & 640 & 1280 & 15.00 & 3.26447 & 2.000 \\
2817 & 1280 & 2560 & 56.79 & 3.26433 & 2.071 \\
5633 & 2560 & 5120 & 239.79 & 3.26426 & 2.000 \\
11265 & 5120 & 10240 & 966.29 & 3.26422 & 1.750 \\
\hline
\end{tabular}

TABlE 6: Convergence study. quadratic variation, Bounded Control. Fully implicit timestepping is applied, using constant timesteps. Parameters are given in Table 5, with $\lambda=0.2873$. Values of $V=E_{t=0, x}^{p^{*}}\left[X(T)-\lambda \int_{0}^{T}\left(e^{r(T-s)} d X^{2}\right)\right]$ Ratio is the ratio of successive changes in the computed values for decreasing values of the discretization parameter $h$. CPU time is normalized. We take the CPU time used for the second test in this table as one unit of CPU time, which uses 353 nodes for $X$ grid and 160 timesteps.

\begin{tabular}{|c|c|c|c|c|c|}
\hline $\begin{array}{c}\text { Nodes } \\
(W)\end{array}$ & Timesteps & $\operatorname{Std}_{t=0, x}^{p^{*}}[W(T)]$ & $E_{t=0, x}^{p^{*}}[W(T)]$ & $\begin{array}{c}\text { Ratio } \\
\text { for } \operatorname{Std}_{t=0, x}^{p^{*}}[W(T)]\end{array}$ & $\begin{array}{c}\text { Ratio } \\
\text { for } E[W(T)]\end{array}$ \\
\hline 177 & 80 & 1.39064 & 3.69771 & & \\
353 & 160 & 1.35723 & 3.69524 & & \\
705 & 320 & 1.34035 & 3.69403 & 1.979 & 2.041 \\
1409 & 640 & 1.33187 & 3.69343 & 1.991 & 2.017 \\
2817 & 1280 & 1.32762 & 3.69313 & 1.995 & 2.000 \\
5633 & 2560 & 1.32549 & 3.69298 & 1.995 & 2.000 \\
11265 & 5120 & 1.32443 & 3.69291 & 2.009 & 2.143 \\
\hline
\end{tabular}

TABLE 7: Convergence study, wealth-to-income ratio case, bounded control. Fully implicit timestepping is applied, using constant timesteps. Parameters are given in Table 5, with $\lambda=0.2873$. Values of $S t d_{t=0, x}^{p^{*}}[X(T)]$ and $E_{t=0, x}^{p^{*}}[X(T)]$ are reported at $(X=0.5, t=0)$. Ratio is the ratio of successive changes in the computed values for decreasing values of the discretization parameter $h$.

if $X(t=0)=0.5,\left(\operatorname{Std}_{t=0, x}^{p^{*}}[X(T)], E_{t=0, x}^{p^{*}}[X(T)]\right)=(1.32443,3.69291)$ and $Q_{-} s t d_{t=0, w}^{p^{*}}[X(T)]=$ 1.49213 from the finite difference solution. Similar to the wealth case, we can see that the control $p$ is a increasing function of time $t$ for a fixed $x$.

Remark 6.2 (Behaviour of the control as a function of time) The optimal strategy for the wealth-to-income ratio case, based on a power law utility function [10] has the property that, for fixed $x$, the control $p$ is a decreasing function of time. In other words, if the wealth-to-income ratio is static, the investor reduces the weight in the risky asset as time goes on [10]. Using the mean quadratic variation criterion, the optimal strategy is to increase the weight in the risky asset if the wealth-to-income ratio is static. 


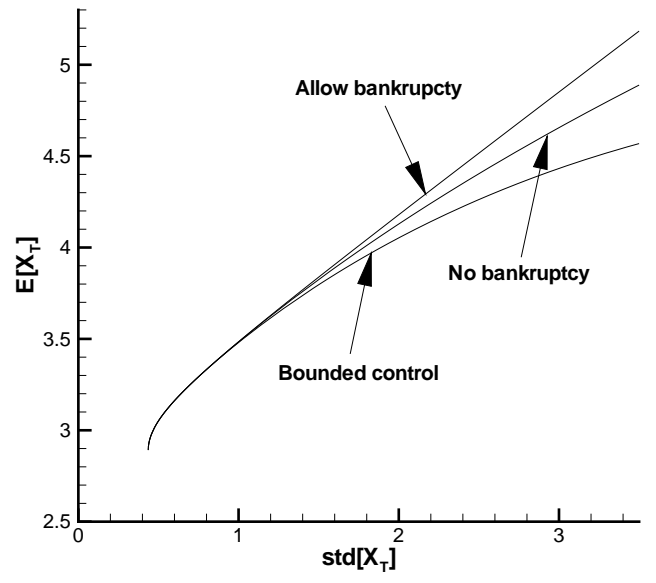

(a) Risk measure: std

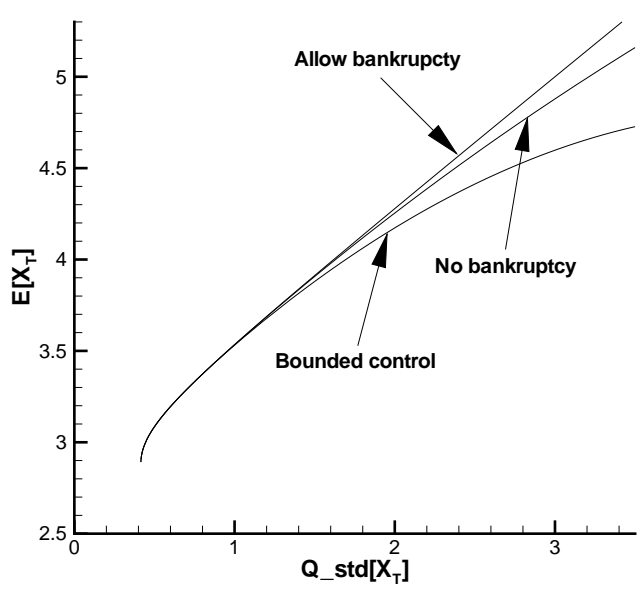

(b) Risk measure: $Q_{-}$std

FIGURE 6: Efficient frontiers (wealth-to-income ratio) for allowing bankruptcy $(\mathbb{D}=(-\infty,+\infty)$ and $\mathbb{P}=(-\infty,+\infty))$, no bankruptcy $(\mathbb{D}=[0,+\infty)$ and $\mathbb{P}=[0,+\infty))$ and bounded control $(\mathbb{D}=[0,+\infty)$ and $\mathbb{P}=[0,1.5])$ cases. Parameters are given in Table 5 . Values are reported at $(W=1, t=0)$. Figure (a) shows the frontiers with risk measure standard deviation. Figure (b) shows the frontiers with risk measure quadratic variation.

\section{Comparison of Various Strategies}

In this section, we compare the three strategies: pre-commitment, time-consistent and quadratic variation strategies. We remind the reader that the pre-commitment solutions are computed using the methods in [29, and the time-consistent strategies are computed using the methods in [30]. The mean quadratic variation results are computed using the techniques developed in this article.

\subsection{Wealth Case}

We first study the wealth case for the three strategies. Figure 8 shows the frontiers for the case of allowing bankruptcy for the three strategies. The analytic solution for the pre-commitment strategy is given in [19],

$$
\left\{\begin{array}{l}
\operatorname{Var}^{t=0}[W(T)]=\frac{e^{\xi^{2} T}-1}{4 \lambda^{2}} \\
E^{t=0}[W(T)]=\hat{w}_{0} e^{r T}+\pi \frac{e^{r T}-1}{r}+\sqrt{e^{\xi^{2} T}-1} \operatorname{Std}(W(T)),
\end{array}\right.
$$

and the optimal control $p$ at any time $t \in[0, T]$ is

$$
p^{*}(t, w)=-\frac{\xi}{\sigma w}\left[w-\left(\hat{w}_{0} e^{r t}+\frac{\pi}{r}\left(e^{r t}-1\right)\right)-\frac{e^{-r(T-t)+\xi^{2} T}}{2 \lambda}\right] .
$$

Extending the results from [5], we can obtain the analytic solution for the time-consistent 


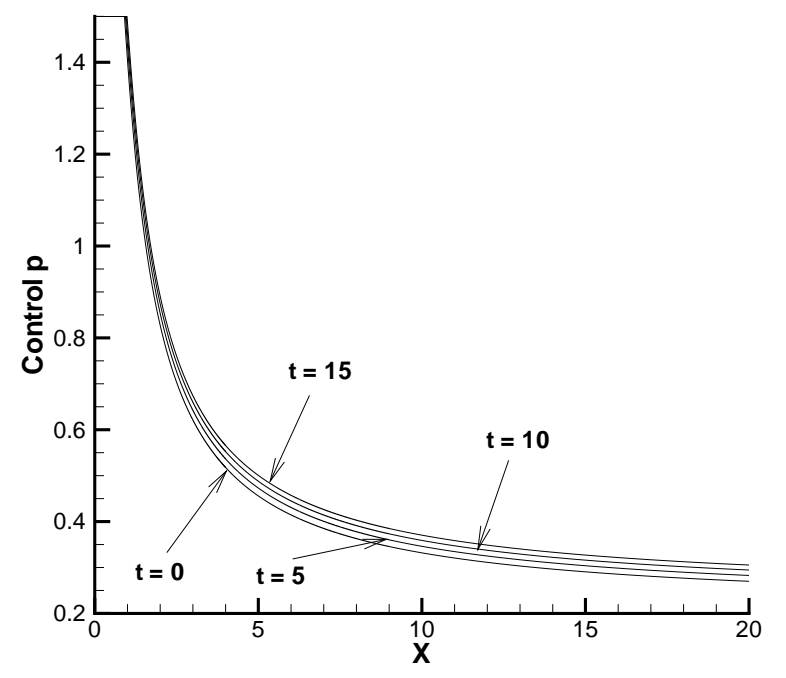

FIgURE 7: Optimal control as a function of $(X, t)$, mean quadratic variation, wealth-to-income ratio with bounded control. Parameters are given in Table 5, with $\lambda=0.2873$. Under these inputs, if $X(t=0)=0.5,\left(\operatorname{St}_{t=0, x}^{p^{*}}[X(T)], E_{t=0, x}^{p^{*}}[X(T)]\right)=(1.32443,3.69291)$ and $Q_{-} s t d_{t=0, x}^{p^{*}}[X(T)]=$ 1.49213 from finite difference solution.

strategy,

$$
\left\{\begin{array}{l}
\operatorname{Var}_{t=0, \hat{w}_{0}}[W(T)]=\frac{\xi^{2}}{4 \lambda^{2}} T \\
E_{t=0, \hat{w}_{0}}[W(T)]=\hat{w}_{0} e^{r T}+\pi \frac{e^{r T}-1}{r}+\xi \sqrt{T} \operatorname{Std}(W(T)),
\end{array}\right.
$$

and the optimal control $p$ at any time $t \in[0, T]$ is

$$
p^{*}(t, w)=\frac{\xi}{2 \lambda \sigma w} e^{-r(T-t)} .
$$

Figure 8 shows that the frontiers for the time-consistent strategy and the mean quadratic variation strategy are the same. This result agrees with the result in [7]. It is also interesting to observe that this control is also identical to the control obtained using a utility function of the form 14

$$
U(w)=-\frac{e^{-2 \lambda w}}{2 \lambda}
$$

Figure 8 also shows that the pre-commitment strategy dominates the other strategies, according to the mean-variance criterion. The three frontiers are all straight lines, and pass the same point at $(\operatorname{Std}(W(T)), E(W(T)))=\left(0, \hat{w}_{0} e^{r T}+\pi \frac{e^{r T}-1}{r}\right)$. At that point, the plan holder simply invests all her wealth in the risk free bond, so the standard deviation is zero.

Remark 7.1 It appears that in general, the the investment policies for time consistent mean variance and mean quadratic variation strategies are not the same. These two strategies do give rise 
to the same policy in the unconstrained (allow bankruptcy) case. When we apply constraints to the investment strategy, the optimal polices are different, but quite close (see the numerical results later in this Section). However, as noted in [7], there exists some standard time consistent control problem which does give rise to the same control. But, as pointed out in [7], it is not obvious how to find this equivalent problem.

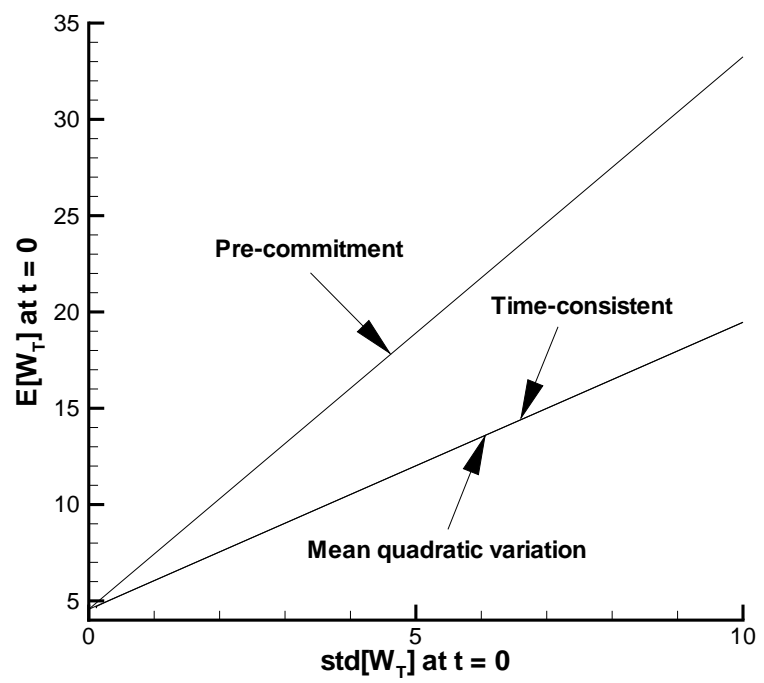

Figure 8: Comparison of three strategies: wealth case, allowing bankruptcy. Parameters are given in Table 2

Figure 9 (a) shows a comparison for the three strategies for the no bankruptcy case, and Figure 9 (b) is for the bounded control case. We can see that the pre-commitment strategy dominates the other strategies. The mean quadratic variation strategy dominates the time-consistent strategy. For the bounded control case, the three frontiers have the same end points. The lower end corresponds to the most conservative strategy, i.e. the whole wealth is invested in the risk free bond at any time. The higher end corresponds to the most aggressive strategy, i.e. choose the control $p$ to be the upper bound $p_{\max }(=1.5)$ at any time. Figure 8 and 9 show that the difference between the frontiers for the three strategies becomes smaller after adding constraints.

Since the frontiers for the time-consistent strategy and the mean quadratic variation strategy are very close for the bounded control case, it is desirable to confirm that the small difference is not due to computational error. In Table 8, we show a convergence study for both time-consistent strategy and mean quadratic variation strategy. The parameters are given in Table 2, We fix $\operatorname{Std}_{t=0, w}^{p^{*}}[W(T)]=5$. Table 8 shows that the two strategies converge to different expected terminal wealth.

It is not surprising that the pre-commitment strategy dominates the other strategies, since the pre-commitment strategy is the strategy which optimizes the objective function at the initial time $(t=0)$. However, as discussed in Section 1 , in practice, there are many reasons to choose a time-consistent strategy or a mean quadratic variation strategy. 


\begin{tabular}{|c|c|c|}
\hline Refine & $\begin{array}{c}\text { Time-consistent } \\
E_{t=0, w}^{p^{*}}[W(T)]\end{array}$ & $\begin{array}{c}\text { Mean Quadratic Variation } \\
E_{t=0, w}^{p^{*}}[W(T)]\end{array}$ \\
\hline 0 & 10.3570 & 10.4337 \\
1 & 10.4508 & 10.5537 \\
2 & 10.5055 & 10.6035 \\
3 & 10.5319 & 10.6273 \\
4 & 10.5448 & 10.6390 \\
5 & 10.55139 & 10.6447 \\
\hline
\end{tabular}

TABLE 8: Convergence study, wealth case, bounded control. Fully implicit timestepping is applied, using constant timesteps. The parameters are given in Table 2. We fix Std $d_{t=0, w}^{p^{*}}[W(T)]=5$ for both time-consistent and mean quadratic variation strategies. Values of $\operatorname{St}_{t=0, w}^{p^{*}}[W(T)]$ and $E_{t=0, w}^{p^{*}}[W(T)]$ are reported at $(W=1, t=0)$. On each refinement, new nodes are inserted between each coarse grid node, and the timestep is divided by two. Initially (zero refinement), for timeconsistent strategy, there are 41 nodes for the control grid, 182 nodes for the wealth grid, and 80 timesteps; for mean quadratic variation strategy, there are 177 nodes for the wealth grid, and 80 timesteps.

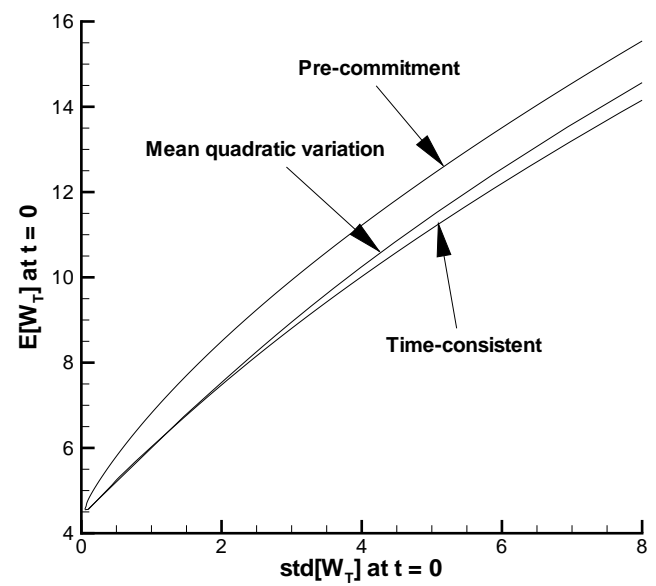

(a) No Bankruptcy

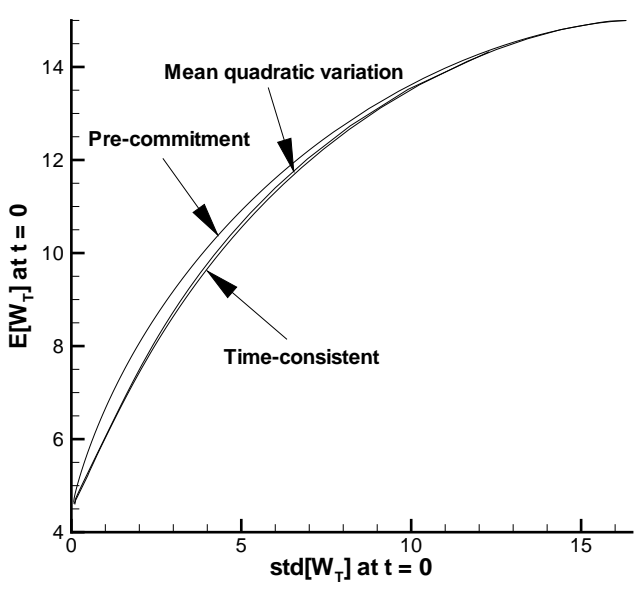

(b) Bounded Control

Figure 9: Comparison of three strategies: wealth case. (a): no bankruptcy case; (b): bounded control case. The parameters are given in Table 2.

In Figure 10, we compare the control policies for the three strategies. The parameters are given in Table 2, and we use the wealth case with bounded control $(p \in[0,1.5])$. We fix $\operatorname{Std}_{t=0, x}^{p^{*}}[W(T)] \simeq$ 8.17 for this test. Figure 10 shows that the control policies given by the three strategies are significantly different. This is true even for the bounded control case, where the expected values for the three strategies are similar for fixed standard deviation (see Figure 9 (b)). Figure 10 (a) shows the control policies at $t=0^{+}$.

We can interpret Figure 10 as follows. Suppose initially $W(t=0)=1$. If at the instant right after $t=0$, the value for $W$ jumps to $W\left(t=0^{+}\right)$, Figure 10 (a) shows the control policies for 


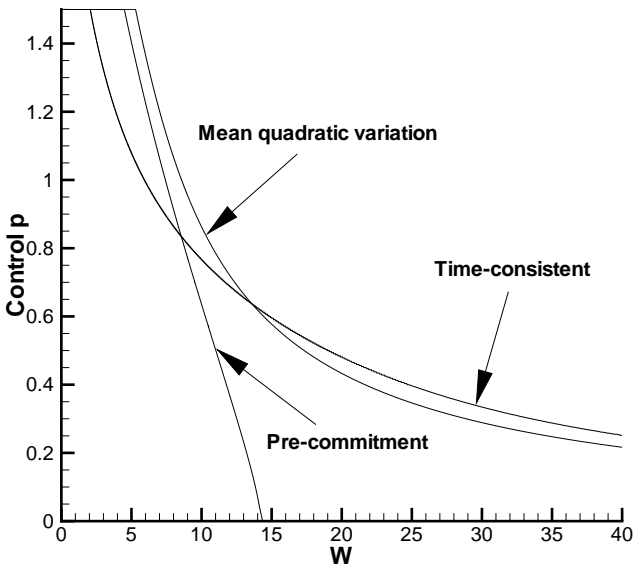

(a)

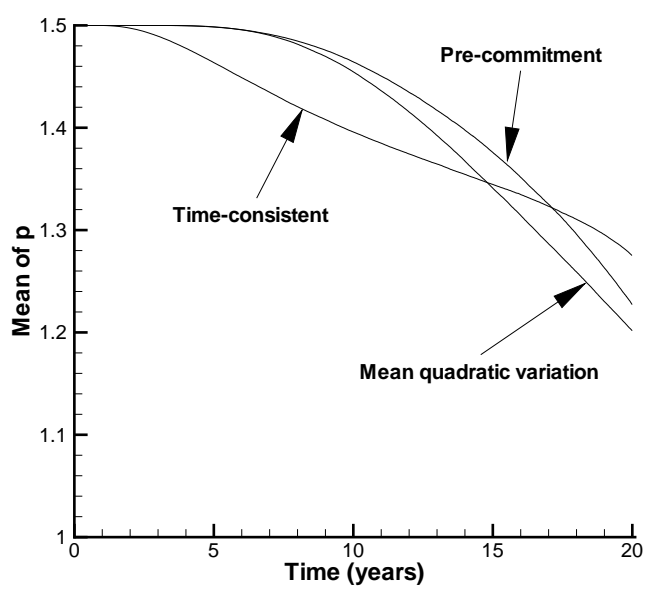

(b)

Figure 10: Comparison of the control policies: wealth case with bounded control $(p \in[0,1.5])$. Parameters are given in Table 2. We fix st $d_{t=0, w}^{p^{*}}[W(T)] \simeq 8.17$ for this test. More precisely, from our finite difference solutions, $\left(\operatorname{St}_{t=0, w}^{p^{*}}[W(T)], E_{t=0, w}^{p^{*}}[W(T)]\right)=(8.17479,12.7177)$ for the mean quadratic variation strategy; $\left(S t d_{t=0, w}^{p^{*}}[W(T)], E_{t=0, w}^{p^{*}}[W(T)]\right)=(8.17494,12.6612)$ for the time-consistent strategy; and $\left(S t t_{t=0, w}^{p^{*}}[W(T)], E_{t=0, w}^{p^{*}}[W(T)]\right)=(8.17453,12.8326)$ for the precommitment strategy. Figure (a) shows the control policies at $t=0^{+}$; Figure (b) shows the mean of the control policies versus time $t \in[0, T]$.

all $W\left(t=0^{+}\right)$. We can see that once the wealth $W$ is large enough, the control policy for the on the value of $\lambda$. Once the target is reached, the investor will not take any more risk and switch all wealth into bonds. However, there is no similar effective target for the time-consistent or the mean quadratic variation cases, so the control never reaches zero. Figure 10 (b) shows the mean of the control policies versus time $t \in[0, T]$. The mean of both policies are decreasing functions of time, i.e. all strategies are less risky (on average) as maturity is approached. We use Monte-Carlo mulations to obtain Figure 10 (b). Using the parameters in Table 2, we solve the stochastic each simulation. We then can obtain the mean of $p$ for each time step.

\subsection{Wealth-to-income Ratio Case}

Figure 11 and 12 shows a comparison for the three strategies for the wealth-to-income ratio case. Figure 11 is for bankruptcy case, Figure 12 (a) is for no bankruptcy case, and Figure 12 (b) is for the bounded control case. Similar to the allowing bankruptcy case, the pre-commitment strategy dominates the other strategies. Note that unlike the wealth case, the frontiers for the 
three strategies do not have the common lower end point. As discussed in Section 6.3, no risk free strategy exists in this case because of the salary risk. Furthermore, since the salary is correlated with the stock index $\left(\sigma_{Y_{1}} \neq 0\right)$, in order to (partially) hedge the salary risk, the most conservative policy is not to invest all money in the bond $(p=0)$ all the time. The three strategies have different views of risk, hence their most conservative investment policies would be different. Therefore, their minimum risks (in terms of standard deviation) are different. Also note that, the frontiers given by the time-consistent strategy and the mean quadratic variation strategy are very close, almost on top of each other.

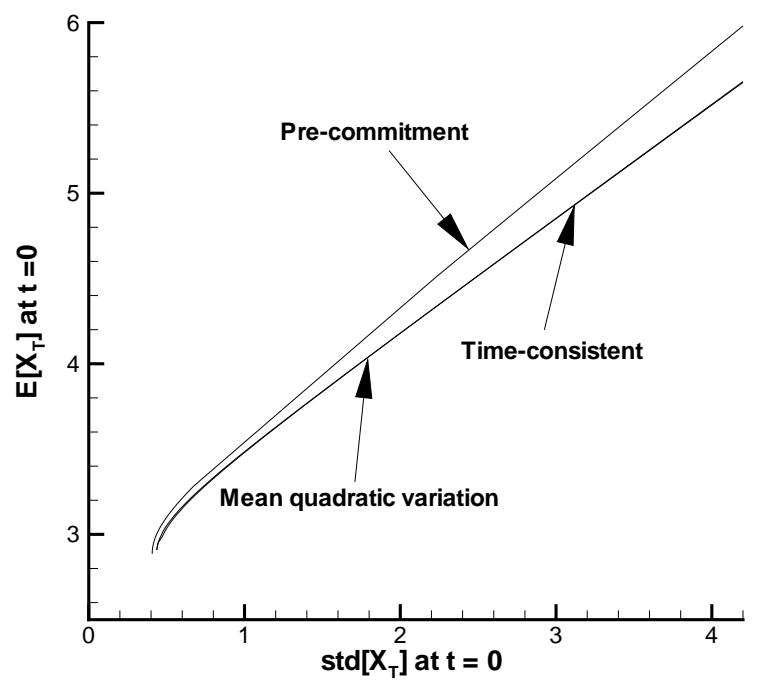

FIGURE 11: Comparison of three strategies: wealth-to-income ratio case, allowing Bankruptcy. Parameters are given in Table 5.

Similar to the wealth case, Figure 13 shows a comparison of the control policies for the three strategies. Parameters are given in Table 5, and we use wealth case with bounded control $(p \in$ $[0,1.5])$. We fix $\operatorname{Std}_{t=0, x}^{p^{*}}[X(T)] \simeq 3.24$ for this test. The comparison shows that although the three strategies have a similar pair of expected value and standard deviation, the control policies are significantly different.

Remark 7.2 (Average strategy) From Remark 6.2. we note that if the wealth-to-income ratio is static, the optimal strategy (under the mean-quadratic-variation criteria) is to increase the weight in the risky asset. This is also observed for the pre-commitment and time consistent policies [29, [30]. Nevertheless, for all three optimal strategies, the mean optimal policy is to decrease the weight in the risky asset as $t \rightarrow T$.

\section{Conclusion}

In this article, we consider three mean variance like strategies: a pre-commitment strategy, a time-consistent strategy (as defined in [5]) and a mean quadratic variation strategy. Although the 


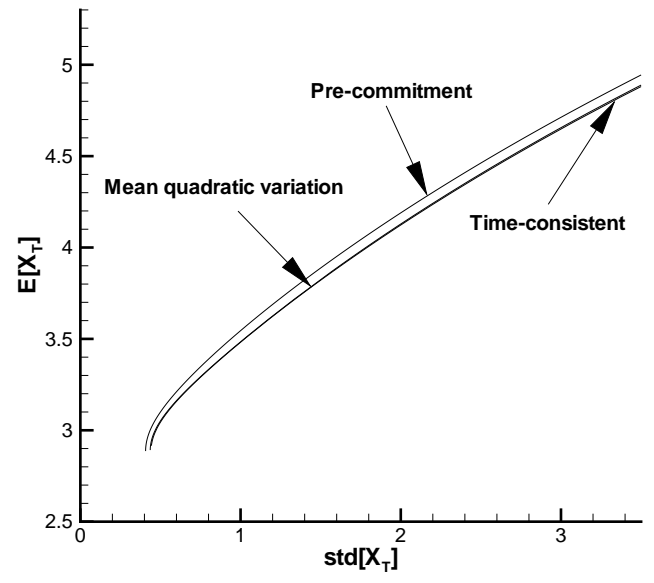

(a) No Bankruptcy

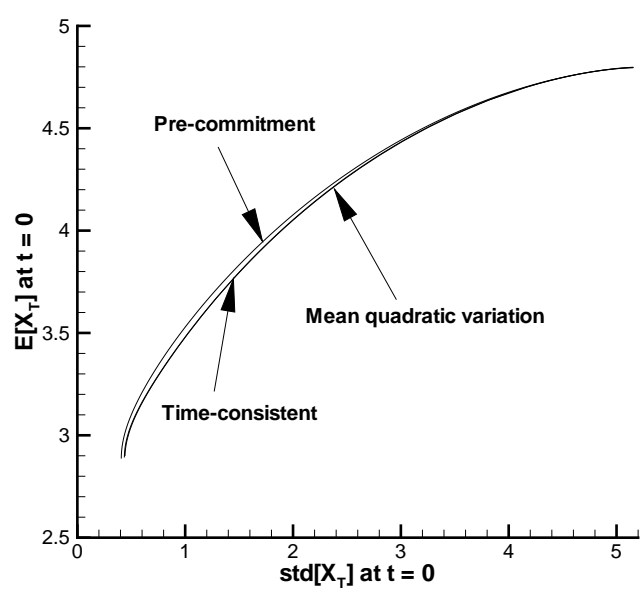

(b) Bounded Control

Figure 12: Comparison of the three strategies: wealth-to-income ratio case. (a): no bankruptcy case; (b): bounded control case. Parameters are given in Table 5 .

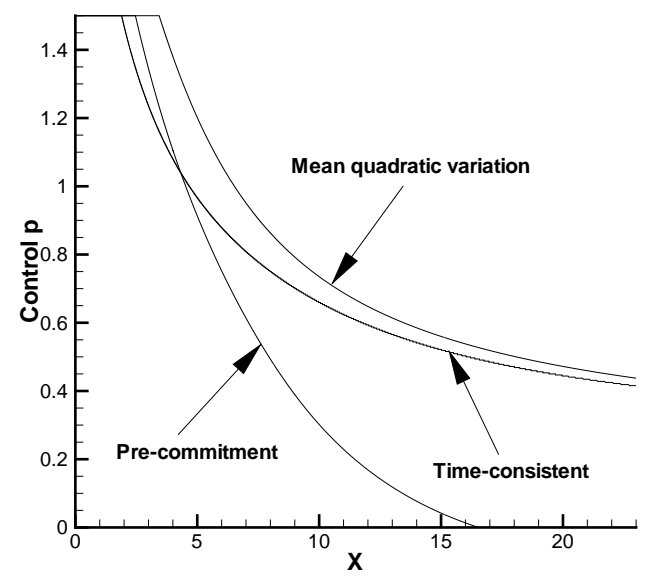

(a)

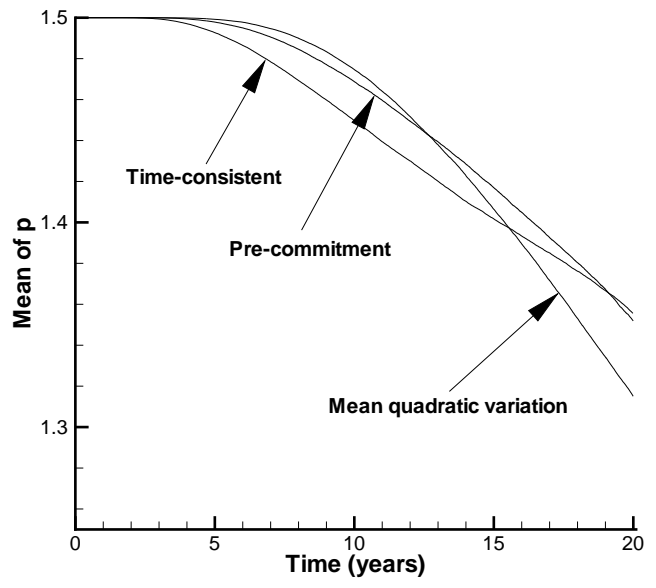

(b)

Figure 13: Comparison of the control policies: wealth-to-income ratio case with bounded control $(p \in[0,1.5])$. Parameters are given in Table 5. We fix st $d_{t=0, x}^{p^{*}}[X(T)] \simeq 3.24$ for this test. More precisely, from our finite difference solutions, $\left(\operatorname{St}_{t=0, x}^{p^{*}}[X(T)], E_{t=0, x}^{p^{*}}[X(T)]\right)=(3.24214,4.50255)$ for the mean quadratic variation strategy; $\left(S t d_{t=0, x}^{p^{*}}[X(T)], E_{t=0, x}^{p^{*}}[X(T)]\right)=(3.24348,4.50168)$ for the time-consistent strategy; and $\left(S t d_{t=0, x}^{p^{*}}[X(T)], E_{t=0, x}^{p^{*}}[X(T)]\right)=(3.24165,4.50984)$ for the precommitment strategy. Figure (a) shows the control policies at $t=0^{+}$; Figure (b) shows the mean of the control policies versus time $t \in[0, T]$. 
pre-commitment strategy dominates the other strategies, in terms of an efficient frontier solution, it is not time-consistent.

In practice, many investors may choose a time-consistent strategy. However, for both precommitment and time-consistent strategies, the risk is only measured in terms of the standard deviation at the end of trading. Practitioners might prefer to control the risk during the whole investment period [16]. The mean quadratic variation strategy controls this risk.

In this paper, we consider two cases for a pension plan investment strategy: the wealth case and the wealth-to-income ratio case. We study three types of constraints on the strategy: the allowing bankruptcy case, a no bankruptcy case, and a bounded control case.

We have implemented numerical schemes for the pre-commitment strategy and the time-consistent strategy in [29, 30]. In this paper, we extend the method in [29] to solve for the optimal strategy for the mean quadratic variation problem. The equation for the value function is in the form of a nonlinear HJB PDE. We use a fully implicit method to solve the nonlinear HJB PDE. It can be shown that our numerical scheme converges to the viscosity solution. Numerical examples confirm that our method converges to the analytic solution where available.

We carry out a comparison of the three mean variance like strategies. For the allowing bankruptcy case, analytic solutions exist for all strategies. Furthermore, the time-consistent strategy and the mean quadratic variation strategy have the same solution. However, when additional constraints are applied to the control policy, analytic solutions do not exist in general.

After realistic constraints are applied, the frontiers for all three strategies are very similar. In particular, the mean quadratic variation strategy and the time consistent mean variance strategy (with constraints) produce very similar frontiers. However, the investment policies are quite different, for all three strategies. This suggests that the choice among various strategies cannot be made by only examining the efficient frontier, but rather should be based on the qualitative behavior of the optimal policies.

\section{A Discrete Equation Coefficients}

Let $p_{i}^{n}$ denote the optimal control $p^{*}$ at node $i$, time level $n$ and set

$$
a_{i}^{n+1}=a\left(z_{i}, p_{i}^{n}\right), b_{i}^{n+1}=b\left(z_{i}, p_{i}^{n}\right), \quad c_{i}^{n+1}=c\left(z_{i}, p_{i}^{n}\right) .
$$

Then, we can use central, forward or backward differencing at any node. Central Differencing:

$$
\begin{aligned}
\alpha_{i, \text { central }}^{n} & =\left[\frac{2 a_{i}^{n}}{\left(z_{i}-z_{i-1}\right)\left(z_{i+1}-z_{i-1}\right)}-\frac{b_{i}^{n}}{z_{i+1}-z_{i-1}}\right] \\
\beta_{i, \text { central }}^{n} & =\left[\frac{2 a_{i}^{n}}{\left(z_{i+1}-z_{i}\right)\left(z_{i+1}-z_{i-1}\right)}+\frac{b_{i}^{n}}{z_{i+1}-z_{i-1}}\right] .
\end{aligned}
$$

Forward/backward Differencing: $\left(b_{i}^{n}>0 / b_{i}^{n}<0\right)$

$$
\begin{aligned}
\alpha_{i, \text { forward/backward }}^{n} & =\left[\frac{2 a_{i}^{n}}{\left(z_{i}-z_{i-1}\right)\left(z_{i+1}-z_{i-1}\right)}+\max \left(0, \frac{-b_{i}^{n}}{z_{i}-z_{i-1}}\right)\right] \\
\beta_{i, \text { forward/backward }}^{n} & =\left[\frac{2 a_{i}^{n}}{\left(z_{i+1}-z_{i}\right)\left(z_{i+1}-z_{i-1}\right)}+\max \left(0, \frac{b_{i}^{n}}{z_{i+1}-z_{i}}\right)\right] .
\end{aligned}
$$




\section{References}

[1] L. Bai and H. Zhang. Dynamic mean-variance problem with constrained risk control for the insurers. Mathematical Methods for Operations Research, 68:181-205, 2008.

[2] G. Barles. Convergence of numerical schemes for degenerate parabolic equations arising in finance. In L. C. G. Rogers and D. Talay, editors, Numerical Methods in Finance, pages 1-21. Cambridge University Press, Cambridge, 1997.

[3] G. Barles, CH. Daher, and M. Romano. Convergence of numerical shemes for parabolic eqations arising in finance theory. Mathematical Models and Methods in Applied Sciences, 5:125-143, 1995.

[4] G. Barles and E. Rouy. A strong comparison result for the Bellman equation arising in stochastic exit time control problems and applications. Communications in Partial Differential Equations, 23:1995-2033, 1998.

[5] S. Basak and G. Chabakauri. Dynamic mean-variance asset allocation. Forthcoming in Review of Financial Studies, 2010.

[6] T.R. Bielecki, Jin H, S.R. Pliska, and X.Y. Zhou. Continuous time mean-variance portfolio selection with bankruptcy prohibition. Mathematical Finance, 15:213-244, 2005.

[7] T. Bjork and A. Murgoci. A general theory of Markovian time inconsistent stochastic control problems. Available at SSRN: http://ssrn.com/abstract=1694759, 2010.

[8] T. Bjork, A. Murgoci, and X. Zhou. Mean variance portfolio optimization with state dependent risk aversion. Working paper, Stockholm School of Economics, 2010.

[9] P. Brugiere. Optimal portfolio and optimal trading in a dynamic continuous time framework. 6'th AFIR Colloquium, Nurenberg, Germany, 1996.

[10] A.J.G. Cairns, D. Blake, and K. Dowd. Stochastic lifestyling: optimal dynamic asset allocation for defined contribution pension plans. Journal of Economic Dynamics and Control, 30:843$877,2006$.

[11] M. Chiu and D. Li. Asset and liability management under a continuous time mean variance optimization framework. Insurance: Mathematics and Economics, 39:330-355, 2006.

[12] G.M. Constantinides. Habit formation: a resolution of the equity premium puzzle. Journal of Political Economy, 98:519-543, 1990.

[13] M. G. Crandall, H. Ishii, and P. L. Lions. User's guide to viscosity solutions of second order partial differential equations. Bulletin of the American Mathematical Society, 27:1-67, 1992.

[14] P. Devolder, M. Bocsch Princep, and Dominguiez Fabian I. Stochastic control of annuity contracts. Insurance: Mathematics and Economics, 33:227-238, 2003.

[15] E. Ekstrom, P. Lotstedt, and J. Tysk. Boundary values and finite difference methods for the single factor term structure equation. Applied Mathematical Finance, 16:253-259, 2009. 
[16] P.A. Forsyth, J.S. Kennedy, S.T. Tse, and H. Windcliff. Optimal trade execution: a mean quadratic variation approach. Submitted to Journal of Economic Dynamics and Control, 2011.

[17] C. Fu, A. Lari-Lavassani, and X. Li. Dynamic mean-variance portfolio selection with borrowing constraint. European Journal of Operational Research, 200:312-319, 2010.

[18] R. Gerrard, S. Haberman, and E. Vigna. Optimal investment choices post retirment in a defined contribution pension scheme. Insurance: Mathematics and Economics, 35:321-342, 2004 .

[19] B. Hojgaard and E. Vigna. Mean variance portfolio selection and efficient frontier for defined contribution pension schemes. Working Paper, Aalborg University, 2007.

[20] M. Leippold, F. Trojani, and P. Vanini. A geometric approach to mulitperiod mean variance optimization of assets and liabilities. Journal of Economic Dynamics and Control, 28:10791113, 2004.

[21] D. Li and W.-L. Ng. Optimal dynamic portfolio selection: Multiperiod mean variance formulation. Mathematical Finance, 10:387-406, 2000.

[22] X. Li and X. Y. Zhou. Continuous time mean variance efficiency and the $80 \%$ rule. Annals of Applied Probability, 16:1751-1763, 2006.

[23] X. Li, X. Y. Zhou, and E. B. Lim. Dynamic mean variance portfolio selection with no-shorting constraints. SIAM Journal on Control and Optimization, 40:1540-1555, 2002.

[24] R.C. Merton. Optimum consumption and portfolio rules in a continuous time model. Journal of Economics Theory, 3:373-413, 1971.

[25] P. Nguyen and R. Portrai. Dynamic asset allocation with mean variance preferences and a solvency constraint. Journal of Economic Dynamics and Control, 26:11-32, 2002.

[26] O.A. Oleinik and E.V. Radkevic. Second Order Equations with Nonnegative Characteristic Form. American Mathematical Society, Providence, 1973.

[27] D.M. Pooley, P.A. Forsyth, and K.R. Vetzal. Numerical convergence properties of option pricing PDEs with uncertain volatility. IMA Journal of Numerical Analysis, 23:241-267, 2003.

[28] S.M. Sundaresan. Intemporally dependent preferences and the volatility of consumption and wealth. Review of Financial Studies, 2:73-89, 1989.

[29] J. Wang and P.A. Forsyth. Numerical solution of the Hamilton-Jacobi-Bellman formulation for continuous time mean varinance asset allocation. Journal of Economic Dynamics and Control, 34:207-230, 2010.

[30] J. Wang and P.A. Forsyth. Continuous time mean variance asset allocation: a time consistent strategy. European Journal of Operational Research, 209:184-201, 2011.

[31] Z. Wang, J. Xia, and L. Zhang. Optimal investment for the insurer: The martingale approach. Insurance: Mathematics and Economics, 40:322-334, 2007. 
[32] X.Y. Zhou and D. Li. Continuous time mean variance portfolio selection: A stochastic LQ framework. Applied Mathematics and Optimization, 42:19-33, 2000. 